\title{
Kinetics of Molecular Diffusion and \\ Self-Assembly: Glycine on $\mathrm{Cu}\{110\}$
}

\author{
Judith B. Rommel* and David Wales \\ Department of Chemistry, University of Cambridge, \\ Lensfield Road, Cambridge, CB2 1EW, United Kingdom. \\ E-mail: jbr36@cam.ac.uk \\ Phone: +44 (0)1223 763872. Fax: +123 (0)123 4445557
}




\begin{abstract}
Nanofabrication and the growth of self-assembled monolayers (SAM) of organic molecules are increasingly important in various industries, including microelectronics and health care. Glycine adsorbed on $\mathrm{Cu}\{110\}$ provides a good model with a rich phenomenological space to explore and understand the self-assembly of more complex amino acids. We focus on (a) the dynamics exhibited by glycine molecules already adsorbed on $\mathrm{Cu}\{110\}$ when diffusing on the metal surface, and (b) the chemical kinetics of how these molecules form clusters, networks, and islands. The stochastic discrete event algorithm we employ can be viewed as a multiscale approach, based on density functional energies and transition barriers. The method covers from the femtosecond time-scale of molecular rotations to the microsecond range of molecular self-assembly. Hydrogen-bonds and van der Waals forces play a crucial role in pattern formation. Investigations of chemical kinetics show that enantiopure, homochiral islands are an intermediate step during the formation process of larger stable racemic, heterochiral islands, especially when two islands merge. At lower temperature, defects stabilise mainly homochiral clusters, and prevent the molecules from synchronising their footprint orientation, in contrast to higher temperature. On the way we solve the long standing puzzle of how the pseudo-centered $(3 \times 2)$ enantiopure clusters can have glide plane symmetry. We end with a comparison to similar amino acids, such as alanine and proline. The results provide insight into mechanisms for fine-tuning the self-organisation of organic molecules on metal surfaces.
\end{abstract}

\title{
Abbreviations: DFT, GLY, ALA, KMC, SAM, TST, STM, RAIRS
}

\section{Introduction}

Nanofabrication plays an important role in various industries, including microelectronics and health care. Biosensors provide one example of such nanodevices, for example, monitoring metabolites, such as blood glucose or lactate, in vivo. ${ }^{1-3}$ Changing the functionality of such 
biosensors to react with different chemical structures requires variations in the electrode coating. Self-assembled monolayers (SAM) of chiral organic molecules, and in particular of amino acids, provide an efficient way to fabricate flexible coatings with a variety of properties. ${ }^{4-8}$ Understanding the fundamentals of spontaneous SAM growth is therefore important to optimise and enhance the electrode coating processes. Many factors can induce self-assembly of organic molecules, including modifications of process conditions, such as $\mathrm{PH}^{9}$ or temperature. ${ }^{10}$ Self-assembly can be coordination-directed, ${ }^{11}$ driven by non-covalent halogen bonding interactions, ${ }^{12}$ or follow a time-dependent protocol using preprogrammed molecules. ${ }^{13}$

Studying the mechanisms and phenomena governing the growth of chiral SAMs over single crystal metal surfaces provides important insights into the spontaneous self-organisation of supramolecular 2D organic networks. ${ }^{14-17}$ Glycine (GLY) adsorbed on $\mathrm{Cu}\{110\}$ is one of the systems that has been more thoroughly investigated over the last two decades. ${ }^{18-24}$ However, although several groups have investigated bonding, structure, and long-range ordering of GLY on $\mathrm{Cu}\{110\}$ using a wide range of experimental ${ }^{18-21}$ and computational tools, ${ }^{22,23}$ the energy barriers between adsorbed GLY conformers, the dynamics of GLY surface diffusion, and the chemical kinetics of cluster, network, and island formation on the surface are still largely unresolved.

GLY undergoes facile dehydrogenation upon adsorption on copper surfaces and it is normally found on the surface in its anionic configuration (glycinate), where the most stable structure is a three-point binding configuration, often denoted $\mu_{3} \cdot{ }^{17,21}$ Although glycine is not intrinsically chiral in the gas phase, when adsorbed on the $\mathrm{Cu}\{110\}$ surface it exhibits the same chiral footprint as alanine (ALA) and proline on this surface (Fig. 1). Hence glycine can be used as a model to understand the assembly of more complex amino acids on $\mathrm{Cu}\{110\} .{ }^{15}$ Fig. 1 shows the model reduction from an atomistic to a coarse-grained representation. The GLY molecules are shown as triangles with oxygen and nitrogen in the corners. The chiral enantiomers of green and blue triangles are red and yellow triangles, respectively.

Apart from exhibiting a mirror symmetry when adsorbed on the $\mathrm{Cu}\{110\}$ surface, similar 


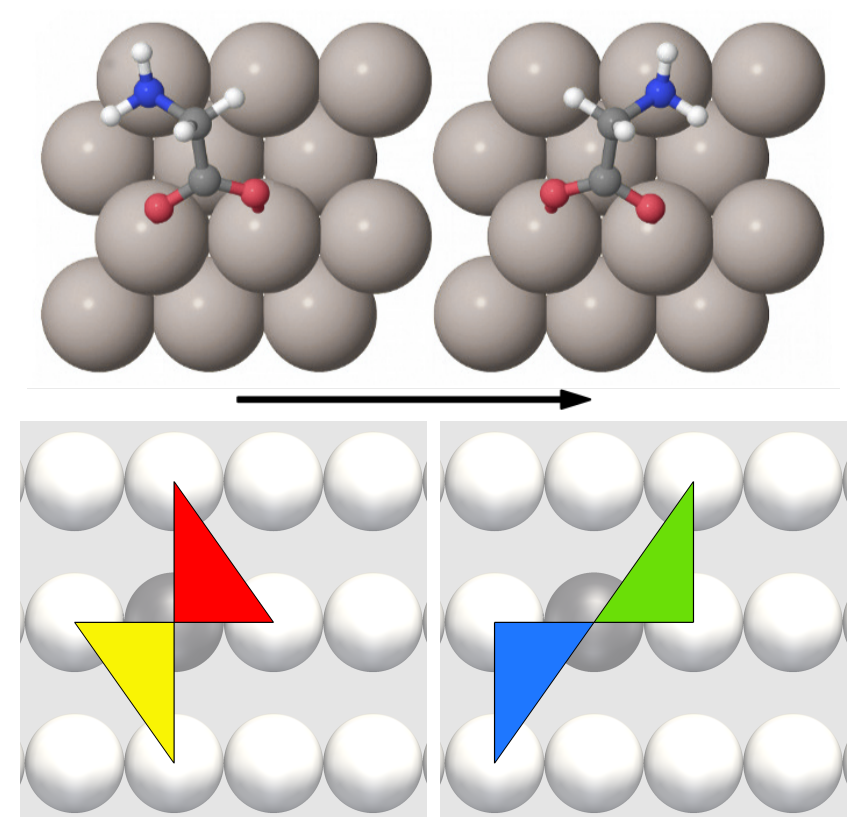

Figure 1: Isolated GLY is achiral, but when adsorbed on $\mathrm{Cu}\{110\}$ as glycinate (top) it can adopt two energetically equivalent orientations (left- and right-handed triangular footprints) that produce footprint chirality. The schematic, coarse-grained representation (bottom) depicts the footprint chirality transition from initial 'left' chirality (red/ yellow) to final 'right' chirality (green/ blue).

to ALA and proline, GLY has two more levels of chirality observable on different length scales: achiral as a single molecule in the gas phase, and a glide plane symmetry as an aggregate in heterochiral domains (Fig. 2). Enantiopure, homochiral domains show a pseudo-centred structure, which we elaborate in more detail in the first section of Results and Discussion. $\mathrm{On} \mathrm{Cu}\{110\}$ adsorption of organic molecules provides a means to control enantioselectivity and the chiral modification of an achiral metal surface. ${ }^{25,26}$ The major scientific challenge of enantiomeric separation is to develop simple, rapid, and sensitive analytical methods, ${ }^{27}$ which can then be extended to enantioselective catalysis. ${ }^{28}$

From this perspective it is crucial to understand how enantiopure and racemic GLY islands form on the metal surface, how molecules mix, and how the island formation is influenced by locally breaking the mirror symmetry of the surface in form of added defects. In contrast to many other self-assembled systems on metal surfaces, the $\mathrm{Cu}\{110\}$ surface has an effect on the directional interactions through covalent bonding, since bonding to 
(a)

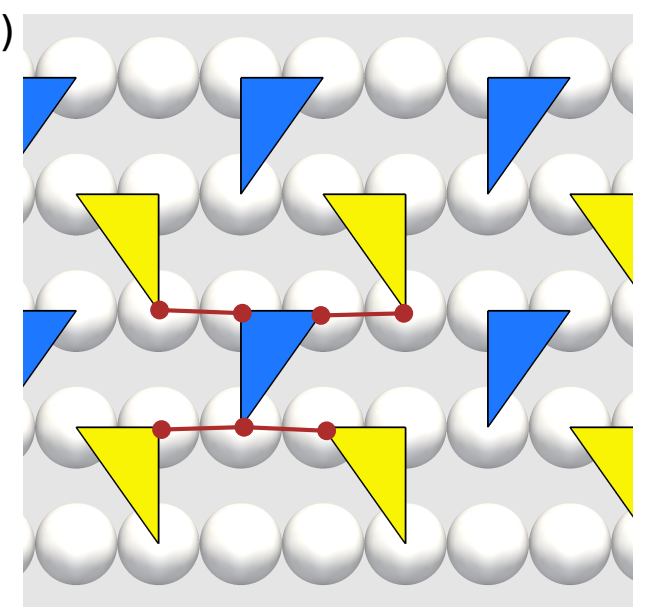

(b)

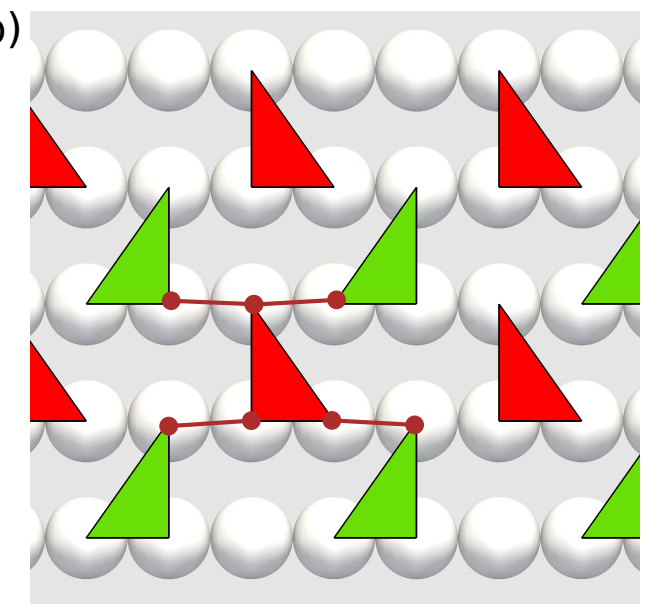

(c)

(d)

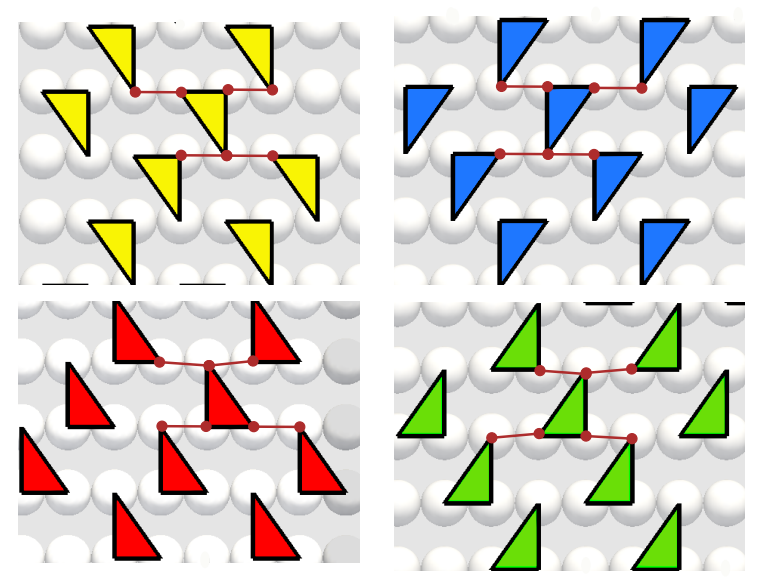

Figure 2: Expected domaines: ${ }^{29}$ (a)-(b) $\mathrm{p}(3 \times 2)$ pg heterochiral domains with glide-line symmetry, both are identical in terms of overall (racemic) chirality (and energy). (c)-(d) four homochiral mirror domains. The nitrogen tips of the top (blue/yellow) and bottom (red/green) triangles point in the same direction and indicate their anisotropy. Marked in red are four possible $\mathrm{NH}-\mathrm{O}$ hydrogen-bonds. 
the copper surface induces dipoles in the adsorbed molecules and creates a positive charge on the nitrogen. ${ }^{30}$ Further driving forces in the chemisorption and the aggregation of GLY molecules on $\mathrm{Cu}\{110\}$ are attractive interactions between the molecules leading to adhesion and cohesion, such as van der Waals forces, and dipole-dipole forces augmented by hydrogenbonding. ${ }^{20}$ We are therefore dealing with a three-dimensional self-assembly process, which provides a rich phenomenological space to explore. From an experimental point of view the chemisorption dynamics is usually understood through indirect assessments. By means of annealing the molecules and scanning tunnelling microscopy (STM) snapshots fixed in time and temperature can be obtained within minutes. ${ }^{31}$ Alternatively, low-energy electron microscopy (LEEM) provides real-time images with spatial resolution of $\mathrm{nm}$ and time scale resolution of seconds. ${ }^{32}$ Techniques to study the dynamics of individual molecules include spin echo methods for the ps time scale, as well as laser pump probing on the fs range. ${ }^{31}$ Computer simulations provide us insight into temperature dependent processes resolved in time on the $\mu$ s time scale, which at present is still challenging for experiments involving multiple GLY molecules on $\mathrm{Cu}\{110\}$.

Based on density functional theory (DFT) ${ }^{33,34}$ energies and transition barriers we construct a coarse-grained multiscale model, where the GLY molecules are represented as triangles with $\mathrm{N}$ and $\mathrm{O}$ atoms defining the corners (Fig. 1). Our approach, a stochastic discrete event algorithm ( $\tau$-leaping), covers the femtosecond time-scales of molecular vibrations and rotations up to events, such as island formation, whose characteristic times are nano- to microseconds. The simulations are highly complementary to experimental investigations of self-assembly and optimal packing for organic molecules on metal surfaces. We combine DFT with transition state theory (TST) ${ }^{35,36}$ to determine the energetics and time scales of the relevant elementary processes, pathways, and rates for footprint chirality conversion. We then investigate the dynamics of molecular diffusion, the chemical kinetics, and the mechanism of cluster, network, and island formation for GLY on $\mathrm{Cu}\{110\}$ with $\tau$-leaping, a Monte Carlo based method, which accounts for the correlations, fluctuations, and spatial distributions of 
the molecules on the metal surface under steady-state conditions. In our investigations we consider a variety of starting conformations. We present results reproducing the experimentally observed cluster and network formation between the anisotropic GLY molecules. We explore whether chirality plays a role, and whether defective surfaces lead to different results. We investigate whether coverage and annealing temperature influence the cluster formation. Varying the energy barriers corresponding to hydrogen-bonds allows us to investigate the role of hydrogen-bonding on molecular network formation. The paper first covers the general methodology of Monte Carlo based molecular kinetics simulations with $\tau$-leaping, and how the femtosecond timescale model based on DFT energies provides the foundation for predictions in the nanosecond range. In the subsequent discussion we present a comparison with experiments before the summary and conclusions.

\section{General methodology}

\section{Molecular Kinetics with $\tau$-Leaping}

We study the dynamics of molecular diffusion and the kinetics of self-assembly of GLY on $\mathrm{Cu}\{110\}$ with Monte Carlo simulations based on a stochastic discrete event algorithm. Monte Carlo based methods have been successfully used to explore a wide range of systems. ${ }^{37-43}$ For example, analysis of diffusion in porous materials and on surfaces reproduced experimental spectra, ${ }^{44-46}$ nucleation or dislocation growth, ${ }^{47-50}$ properties of supercooled liquids and glasses,${ }^{51-53}$ surface reactivity, ${ }^{54}$ peptide detachment processes,${ }^{55}$ semiclassical dynamics, ${ }^{56}$ and predictions of chemical kinetics. ${ }^{57-63}$ Discrete path sampling ${ }^{64-67}$ and Markov state models ${ }^{68-72}$ represent alternative ways to address master equation dynamics via kinetic transition networks. In contrast to molecular dynamics simulations, which are limited by the femtosecond time scale of atomic vibrations, stochastic kinetic Monte Carlo (KMC) ${ }^{73-77}$ simulations facilitate direct modelling of rare events that occur on time scales of milliseconds or longer. ${ }^{52,78,79}$ The multiscale character of this approach provides access to a wide range of 
temporal and spatial scales. ${ }^{80} \mathrm{KMC}$ and its variants are well established simulation methods for studying complex chemistry at the nanoscale. ${ }^{37-43,76,79,81-90}$ We employ a local KMC model, where one molecule moves at an instance in time, and extend it with a method known as $\tau$-leaping, based on the Gillespie algorithm. ${ }^{91}$ The extension offers an efficient choice of the next molecular move and the most probable time step.

Our kinetic model falls into the category of discrete-space and continuous-time Markov processes. The distinct minima and transition states calculated for the energy landscape of GLY on $\mathrm{Cu}\{110\}$ are used to determine the equilibrium reaction and transition rates for the discrete state-to-state dynamics of single molecules. The system passes through a sequence of states $\left\{x_{t_{k}} \in \mathcal{X}\right\}$, a Markov chain, drawn from a model dependent state space $\mathcal{X}$ at times $\left\{t_{0}<t_{1}<\cdots<t_{k}<\ldots\right\}$. The state space $\mathcal{X}=\left\{X_{n}\right\}$ is described by the various local energy minima that the GLY molecules can occupy on the copper surface. Each GLY molecule diffuses along or across the $\mathrm{Cu}\{110\}$ rows from one minimum, $X_{i}$, to another, $X_{j}$, via a fixed set of $n=10$ possible moves. The moves were identified via DFT calculations (see next section) ${ }^{92}$ and remain the same over time, unless neighbouring atoms block a move (Fig. 3).

According to the reported RAIRS (Reflection-Absorption IR Spectroscopy) results, GLY adopts a two-point binding arrangement $\left(\mu_{2}\right)$ at low temperature and low coverage, but converts readily to an overall more stable $\mu_{3}$ footprint on increasing coverage and surface temperature. ${ }^{17,21}$ Since we performed most of our simulations at medium to low coverage (about 0.15 to 0.4 monolayers) and a $\mu_{3}$ conformer is energetically favourable compared to $\mu_{2}$, the GLY molecules in our model lie flat and are always bonded at three points to the surface. At high coverage the space on the surface might limit such a packing. Moreover, the GLY molecules never fully desorb. Therefore the molecular moves are limited to jumps to next-nearest neighbour sites. The escape rates from $X_{i}$ to $X_{j}$, with $i, j \in\{1, \ldots, n\}$, are based on TST, which is appropriate since the dynamics are mainly governed by rare events between states on the potential energy surface, and the vibrational motions occur on a much 


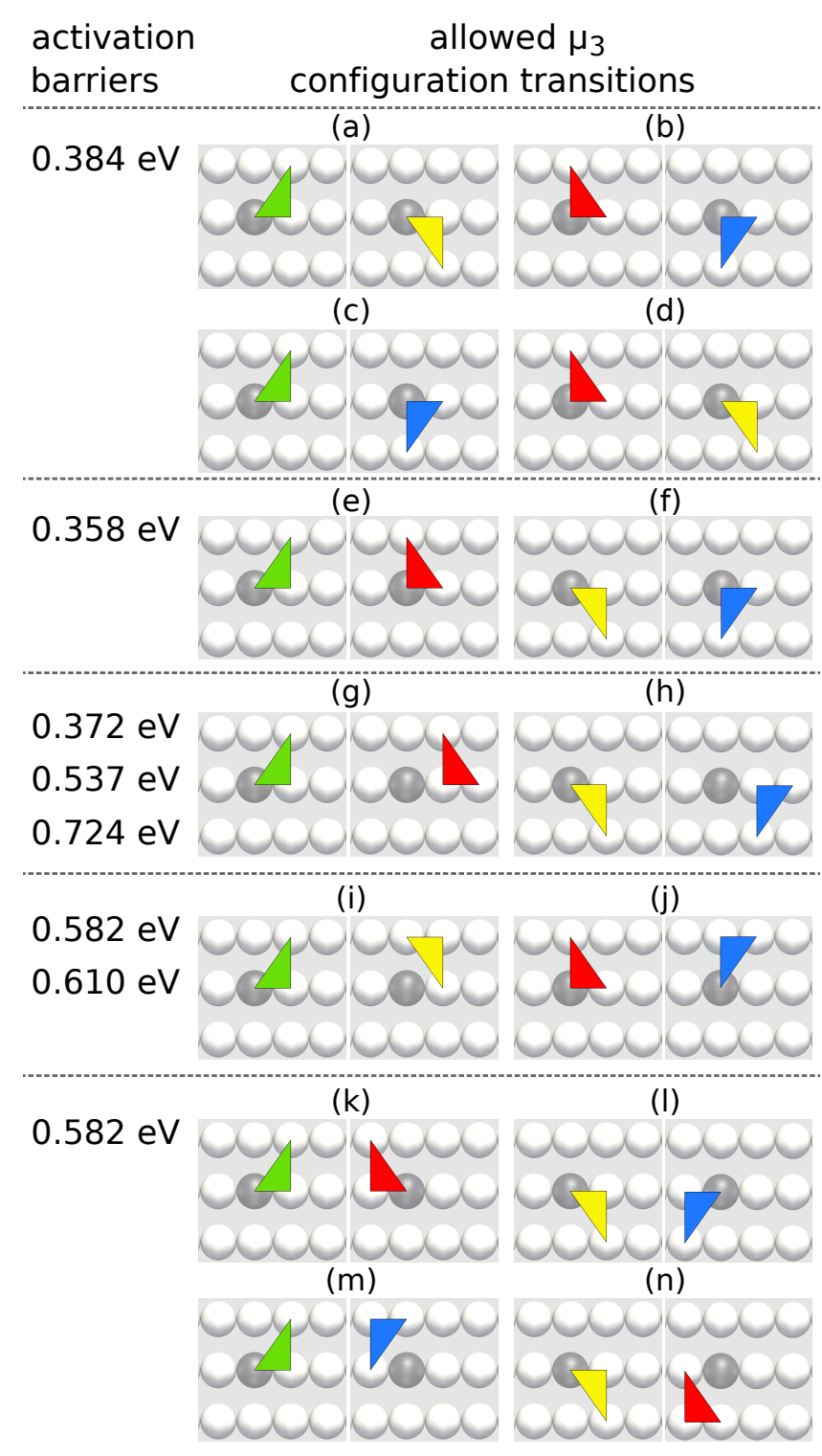

Figure 3: Summary of pathways for the four energetically equivalent $\mu_{3}$ configuration transitions of GLY molecules (initial and final states represented by triangles). ${ }^{92}$ Green and blue, and red and yellow triangles have same footprint chirality, but different surface orientation.

faster time scale. The explicit formula reads

$$
q_{i j}=A e^{-\Delta E_{i j} / k_{B} T},
$$

where $k_{B}$ is the Boltzmann constant and $T$ is the temperature of the system. The prefactor $A=10^{-13} \mathrm{~s}^{-1}$ was chosen to obtain rates on the femtosecond timescale. The energy difference 
$\Delta E_{i j}$ between minimum $X_{i}$ and the transition state connecting it to minimum $X_{j}$ is equal to the reverse transition barrier $\Delta E_{j i}$.

The Markov chain $\left\{x_{t_{k}}\right\}$ is associated with an inhomogeneous Poisson process and the rate $Q(t)$, which is used to determine a sequence of waiting times $\left\{\Delta t_{k}\right\}$ for the intervals between the transitions. Since previous DFT calculations demonstrated that GLY exists on $\mathrm{Cu}\{110\}$ as a single adlayer structure, ${ }^{93}$ we assume that the molecules do not overlap and we deal with independent energy minima. Additionally, theoretical and experimental analysis of photoelectron spectra demonstrated chemically distinct behaviour between diglycine and GLY adsorbed on $\mathrm{Cu}\{110\},{ }^{24,94}$ which justifies our focus on a single adlayer. Moreover, the formation of a peptide bond between GLY molecules would involve a substantially higher barrier than for self-assembly, similar to what has been found in studies of adenine,${ }^{95}$ serine,${ }^{96}$ and ALA. ${ }^{97,98}$ Because the moves from one minimum to another are independent, and the energy minima are uncorrelated, the sum of rates

$$
Q\left(t_{k}\right)=\sum_{X_{j} \in \mathcal{X}} q_{i j}
$$

gives the rate for the overall process at the current time step $t_{k}$. From these rates we define transition probabilities as

$$
p_{i j}=\frac{q_{i j}}{Q} \Rightarrow \sum_{X_{j} \in \mathcal{X}} p_{i j}=1 .
$$

The transition probabilities serve as input to calculate waiting times $\left\{\Delta t_{k}\right\}$ for the moves of each molecule on the surface. We generate waiting times from a Poisson distribution, $\Delta t_{k}=-(1 / Q) \ln u$ with random number $u \in(0,1]$, rather than just taking the mean of the times directly obtained from the transition probabilities. The sum of waiting times for each step taken in the algorithm is a more meaningful quantity to compare with experiments, rather than probabilities. So far we have described the dynamics of a single GLY molecule on the surface. For several interacting surface molecules we apply the $\tau$-leap algorithm. ${ }^{91}$ At each step the most likely subsequent change in the distribution of adsorbed GLY molecules 
is determined from the waiting time $\tau$. We therefore randomly choose - again from a Poisson distribution - the triangle with the lowest waiting time, since it is the one most likely to move next.

The GLY molecules interact with each other due to van der Waals forces and dipoledipole forces augmented by hydrogen-bonding. ${ }^{20,30}$ Experimental studies with low-energy electron diffraction, near-edge X-ray absorption fine structure spectroscopy, X-ray photoelectron spectroscopy, and temperature-programmed desorption for GLY on $\mathrm{Cu}\{531\}$ found that pair formation is induced by hydrogen-bonds between adjacent molecules. Similarly, diglycine molecules on $\mathrm{Cu}\{110\}$ were connected through a network of hydrogen bonds. ${ }^{99}$ The possibilities for establishing hydrogen-bonds with neighbouring atoms are $\mathrm{NH}-\mathrm{O}$ or $\mathrm{CH}-\mathrm{O}$ connections. The $\mathrm{N}$ atom exhibits stronger electronegativity compared to the $\mathrm{C}$ atom, so we expect that the $\mathrm{NH}-\mathrm{O}$ hydrogen bond is much stronger than the $\mathrm{CH}-\mathrm{O}$ bond. ${ }^{100}$ Hence we focus on the four NH-O hydrogen bonds a GLY molecule can form with its direct neighbours (Fig. 2). We investigate a variety of interaction strengths in the form of different barrier heights $\Delta E_{H}$ of $0.075 \mathrm{eV}, 0.1 \mathrm{eV}, 0.125 \mathrm{eV}$, and $0.15 \mathrm{eV}$. Previous estimates predicted the contribution of hydrogen bonds as a few tenths of an eV, $0.22 \mathrm{eV}$ for $\mathrm{NH}-\mathrm{O} .{ }^{100}$ Establishing an interaction between two GLY molecules reduces the barrier used in the rate calculation by $-\Delta E_{H}$, while breaking a bond increases it. Test calculations confirmed that the barrier for establishing two interactions with the same molecule is much higher than with two different molecules. The introduction of a repulsive constant of $2 \Delta E_{H}$ between molecules that are already connected hampered the establishment of a second interaction between them and was essential for the appearance of the experimentally observed heterochiral pattern in our results. To be precise the rate for establishing a bond is

$$
q_{i j}=A e^{-\left(\Delta E_{i j}-m \Delta E_{H}\right) / k_{B} T}, \quad m \in\left\{0, \ldots, \operatorname{dim}\left(\mathcal{M}_{i} \subset \mathcal{X}\right)\right\}
$$

where $m$ refers to the number of $\mathrm{NH}-\mathrm{O}$ interactions a molecule can have with its four 
potential neighbours in the neighbourhood set $\mathcal{M}_{i}=\left\{X_{i}^{1}, \ldots, X_{i}^{4}\right\}$. If a molecule already has an interaction with a neighbour $X_{i}^{l}, l \in 1, \ldots, 4$ then the rates with increased barriers to establish a second interaction with the same molecule are

$$
q_{i j}=A e^{-\left(\Delta E_{i j}-m \Delta E_{H}+2 \Delta E_{H}\right) / k_{B} T}, \quad m \in\left\{0, \ldots, \operatorname{dim}\left(\mathcal{M}_{i} \subset \mathcal{X}\right)\right\} .
$$

Since the molecular interactions influence the barrier heights $\Delta E_{j i}$ through $\Delta E_{H}$, and subsequently the transition rates, the waiting times in the $\tau$-leap algorithm vary from step to step. The effects of small-scale interactions between neighbouring molecules on the collective mechanism of dynamics and kinetics of GLY on $\mathrm{Cu}\{110\}$ are therefore covered by our probabilistic model.

An alternative approach to the $\tau$-leap algorithm, i.e., to model correlated and simultaneous molecular moves, is provided by a coarse-time-step method for global updating. ${ }^{101}$ Our local implementation without cooperative or simultaneous moves has the advantage that the change in configuration state between the time steps is spatially localised. Hence during simulation runs only updates concerning the molecular interaction topology or site occupation changes up to the second nearest neighbours after a molecule moves are required. We implemented efficient look-up tables and updating strategies for the list of possible moves. ${ }^{102}$

We investigate the chemical kinetics on a $20 \times 20$ atom $\mathrm{Cu}\{110\}$ surface with periodic boundary conditions, which mimics a larger (infinite) slab. Usually, experimental GLY clusters cover an area of more than $100 \AA^{2}$, which is reflected in the infinite range due to periodic boundary conditions. A length scale involving a small fraction of the $100 \AA^{2}$ leads to more detailed insights about the mechanisms of island formation.

To analyse the long-range behaviour of the clusters we assign each molecule a centre of mass (COM), based on the positions of the oxygen and nitrogen atoms of each molecule, which sit in the corners of the triangles used in the kinetic model. We calculate the Euclidean distance between each COM over time. To investigate the mechanisms of island formation 
and the role of chirality in detail we simulated ten different starting configurations of 0.1275 , $0.135,0.165$, and 0.2175 monolayers at $500 \mathrm{~K}$. To gain insight into the effect of warming the surface we compare results over a wide temperature range, from $350 \mathrm{~K}$ to $500 \mathrm{~K}$. In experiments GLY molecules are dosed above $408 \mathrm{~K}$ or deposited at room temperature followed by annealing at moderate $(420 \mathrm{~K})$ temperature. ${ }^{17}$ The DFT energy barriers for the motion along and across the top layer copper atom rows are substantial. Thus, the annealing temperature required for effective thermal activation of molecular self-assembly may be quite high. The role of coverage during the island formation process is explored with 100, 50, and 20 GLY molecules on the $20 \times 20$ copper surface, i.e., $0.75,0.375$, and 0.15 monolayers. Coverage is calculated as the number of atoms in GLY molecules on the surface divided by the number of top layer surface atoms. By defining areas on the metal surface where GLY molecules are excluded, we mimic defects on the metal and predictions of the role of defects during the island formation become feasible. In the following sections we present figures for the most interesting results. A full set of images and results is provided in the Supporting Information (SI).

\section{Short Time-Scale Diffusion and Energy Barriers}

We focus on GLY adsorbed as glycinate in a $\mu_{3}$ footprint, with both oxygens and nitrogen bound to the copper surface on close-packed [110] rows, which is the most stable adsorbed configuration obtained in experiments. ${ }^{17,21}$

For a coherent narrative we present calculational details of the DFT simulations ${ }^{92}$ and how they form the basis of our discrete kinetic model. The DFT energy calculations were performed with CASTEP, ${ }^{103}$ a plane-wave, periodic boundary conditions DFT code. The adsorbate was modelled as a single glycinate adsorbed on a $\mathrm{Cu}\{110\}-(3 \times 2)$ cell. The surface was modelled by a five layer slab of $(3 \times 2)$ periodicity, with the top two layers allowed to relax during the geometry optimisations and transition state calculations. The Brillouin zone was sampled by a $(4 \times 4 \times 1)$ Monkhorst Pack k-point grid. ${ }^{104}$ As for previous related 
work, ${ }^{105}$ the Perdew-Burke-Ernzerhof (PBE) exchange-correlation functional ${ }^{106}$ and Vanderbilt ultrasoft pseudopotentials ${ }^{107}$ with a kinetic energy cutoff of $340 \mathrm{eV}$ was used. Both single-ended and double-ended transition state search calculations were performed using a combination of CASTEP Linear Synchronous Transit (LST)/ Quadratic Synchronous Transit (QST) method ${ }^{108}$ and hybrid eigenvector-following as coded in the OPTIM program. ${ }^{109-111}$

GLY, as ALA, adopts a $\mu_{3}$ footprint and a heterochiral (in terms of surface chirality) arrangement ${ }^{22,29}$ in the $(3 \times 2)$ phase. Three different $\mu_{3}$ conformers with the same footprint, but different C-C-N backbone torsional angles, have been identified (Fig. 4).

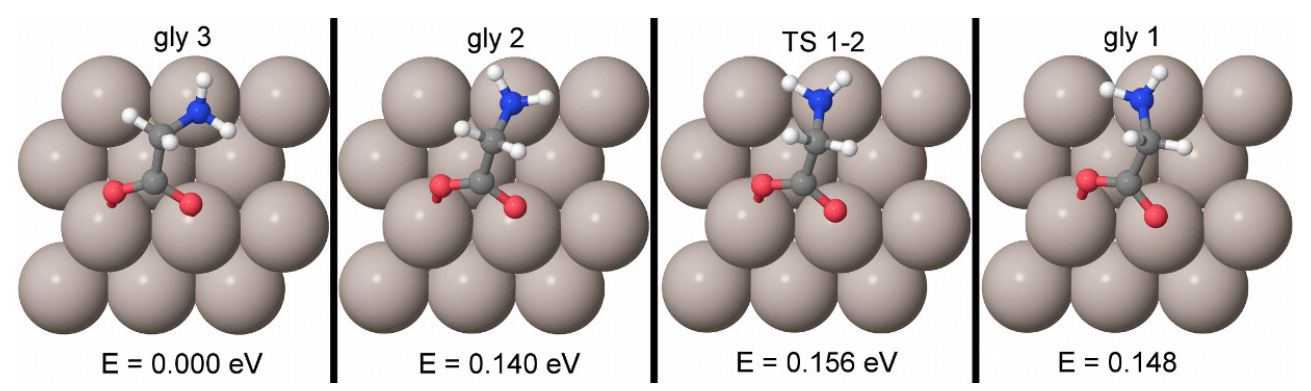

Figure 4: gly 3 , gly 2 and gly 1 are three conformers of glycine adsorbed on $\mathrm{Cu}\{110\}$ in a $\mu_{3}$ binding configuration. ${ }^{92}$ TS 1-2 is the transition state structure between gly 1 and gly 2 with a barrier height of only $8 \mathrm{meV}$. The unidentifiable TS structure between gly 2 and gly 3 , suggests that the energy barrier between these two conformers is lower than the precision available in the DFT calculations (about $5 \mathrm{meV}$ ).

The stronger hydrogen-bonding between one of the $\mathrm{N}-\mathrm{H}$ bonds and the adsorbed oxygen determines the overall greater stability of the gly 3 conformer (the $\mathrm{H}-\mathrm{O}$ distance $\mathrm{d}(\mathrm{O}-\mathrm{H})$ is $2.43 \AA)$ compared to gly $1(\mathrm{~d}(\mathrm{O}-\mathrm{H})=2.73 \AA)$ and gly $2(\mathrm{~d}(\mathrm{O}-\mathrm{H})=2.51 \AA)$ since other structural parameters, such as the lengths of the C-C and C-N bonds, as well as the angle of the C-C-N backbone are very similar among the three conformers. The barrier height for the TS between gly 2 and gly 3 seems to be comparable to the precision available in the DFT calculations $(\sim 5 \mathrm{meV})$.

As expected from experiments, where the production of an ordered $(3 \times 2)$ adlayer requires annealing the surface at about $450 \mathrm{~K},{ }^{17}$ GLY diffusion is found to be a thermally activated process with substantial energy barriers. The investigations are based on the assumption, 
that in order to diffuse parallel to the $\mathrm{Cu}\{110\}$ steps, GLY molecules change footprint chirality after each step, see Fig. 1. Either the amino or the carboxylate group remains bonded to the same site, while the other group changes its binding configuration. The premise that a molecule breaks the minimum number of bonds during a surface diffusion step is justified by both STM and RAIRS observations, which agree that the adsorbate is never bound to the surface via a single $\mathrm{Cu}-\mathrm{O}$ or $\mathrm{Cu}-\mathrm{N}$ bond. Therefore, although not a priori impossible, a step that would include a TS with a single molecule-to-surface bond would probably be energetically unfavourable.

The molecules diffuse along the close-packed $\mathrm{Cu}\{110\}$ rows (Fig. 5) via four different rearrangement pathways while changing from left to right footprint chirality. The mechanism for the first diffusion path involves the $\mathrm{NH}_{2}$ group of GLY hopping from an atop site to an adjacent atop site passing through a transition state (TS 1, Fig. 5) in which the nitrogen atom is on a bridge site. The second mechanism involves synchronous shifting along the [110] direction of the two $\mathrm{O}$ atoms. At the transition state (TS 2, Fig. 5), the molecule is in a pseudo- $\mu_{3}$ configuration, with an $\mathrm{O}$ atom bonded on a bridge site and the other $\mathrm{O}$ in an off-atop position at a slightly longer distance from the surface with respect to the first oxygen. The third diffusion pathway can be described as a $180^{\circ}$ rotation of the OCO group around one of the bonded $\mathrm{O}$ atoms. At the TS 3 and TS-4, Fig. 5, the molecule is in a twopoint binding configuration $\left(\mu_{2}\right)$ with the $\mathrm{N}$ atom bonded on an atop site and an $\mathrm{O}$ atom on a long-bridge site between two steps. The $\mathrm{NH}_{2}$ hopping mechanism (path 1) has the lowest activation energy $(0.358 \mathrm{eV})$, but the OCO shifting mechanism (path 2) has a very similar energy barrier (only about $15 \mathrm{meV}$ higher), so it is likely that, at the surface temperatures generally used in experiments, GLY surface diffusion could involve both pathways. The OCO rotation mechanism (path 3) has an energy barrier $0.18 \mathrm{eV}$ higher than the other two other diffusion pathways, so it is less probable that GLY diffusion will involve the breaking of single $\mathrm{Cu}-\mathrm{O}$ bond with the molecule passing through a $\mu_{2}$ TS configuration. The same reasoning holds for path 4 . 


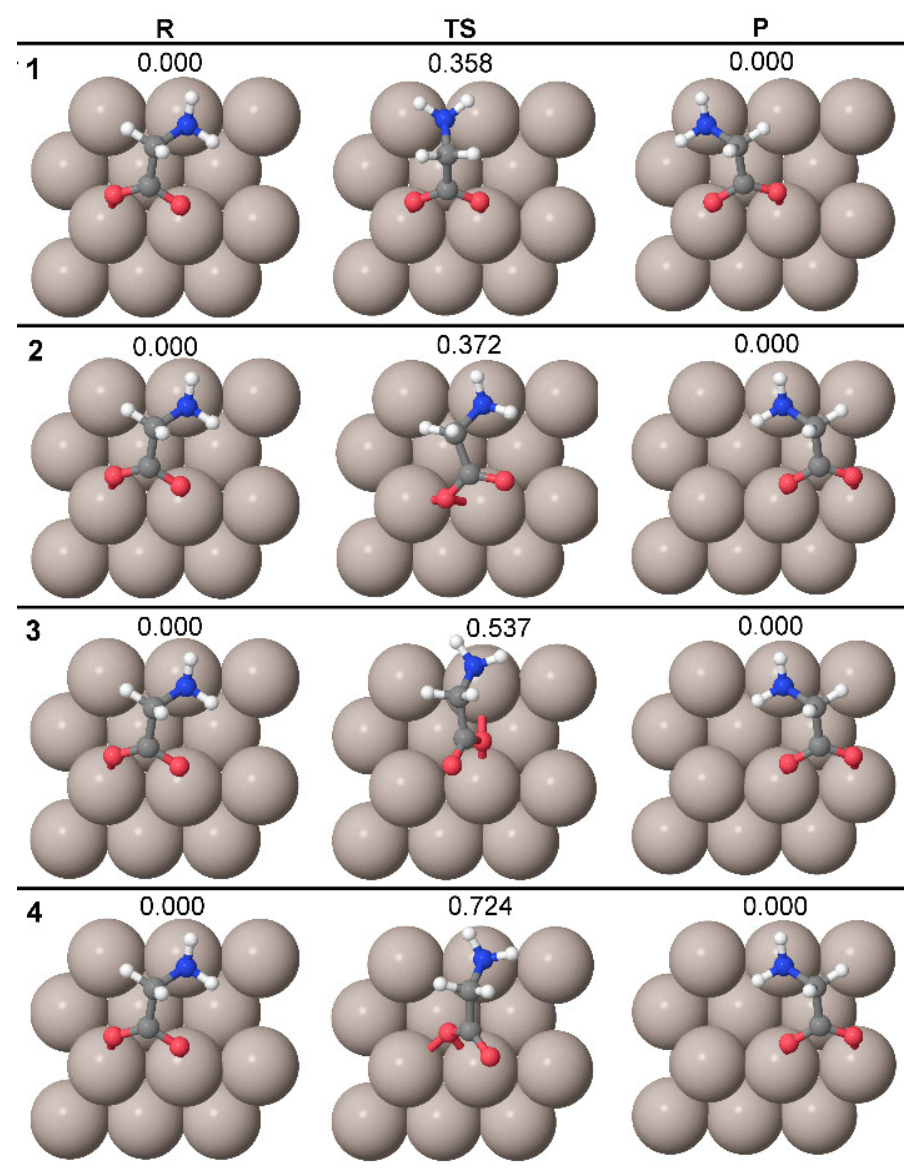

Figure 5: Diffusion along the $\mathrm{Cu}\{110\}$ rows: ${ }^{92}$ paths 1 to 4 , see numbers on the left, with initial geometry ( $\mathrm{R}$ or reactant) on the left panel, the transition state structure (TS) in the centre, and final product $(\mathrm{P})$ on the right. Above each configuration the energy difference is given in $[\mathrm{eV}]$.

The motion of glycine across the close-packed rows, in the [001] and equivalent directions, is an essential part of the surface diffusion process. Through these motions the molecules can form hydrogen-bonds between the carboxylate and the amino groups in molecules occupying alternating rows. In Fig. 6 path 5 describes glycine changing from a $\mu_{3}$ configuration to an intermediate standing configuration by breaking the $\mathrm{Cu}-\mathrm{N}$ bond on one row. The barrier is $0.384 \mathrm{eV}$ and the transition state configuration is very close in geometry and energy to the stable $\mu_{2}$ intermediate $(\mathrm{P})$, in which the molecule binds with the surface through the oxygen atoms of the OCO group only. An internal N-H-O hydrogen-bond is formed, which helps to reduce the energy barrier for this step. From the $\mu_{2}$ intermediate configuration $(0.332 \mathrm{eV})$ the 
$\mathrm{NH}_{2}$ group can adsorb back on an adjacent row. Fig. 3 (a)-(d) summarises the diffusion step and its four possible initial and final configurations (depending on the initial footprint, left or right oriented). By binding two different close-packed rows at the end of the $\mathrm{NH}_{2}$ 'jump' a molecule can change both footprint chirality and orientation at the same time. However, since the carboxylic groups remain bound to the same site, a repetition of multiple jumps will prevent the molecule diffusing across the rows.

Diffusion in a direction perpendicular to the close-packed rows requires the motion of both binding groups: in Fig. 6 path 6 GLY, initially in the $\mu_{3}$ minimum energy configuration, undergoes a concerted translation and rotational motion that shifts its centre of mass towards the close-packed row, bound to the amino group. The associated barrier is only about $0.4 \mathrm{eV}$. The molecule ends in a higher energy $\mu_{3}$ configuration, in which the OCO is on a long-bridge site, binding with two adjacent close-packed rows. The $\mathrm{NH}_{2}$ group is still bound to the same copper atom, but moves from an atop site to an off-top site. This path 6 is the first step of the mechanism that GLY molecules use to move across the close-packed rows. It continues either via path 7 or 8 . In Fig. 6 path 7, the GLY molecule hops across the ridge site landing in a $\mu_{3}$ long-bridge configuration, which is the mirror image of the initial structure. By repeating step 6 , but in the opposite direction the molecule can then shift back to a global minimum configuration.

Alternatively, Fig. 6 path 8, the molecule switches the binding site of the amino group from a long-bridge $\mu_{3}$ configuration to a vertical $\mu_{2}$ geometry through a transition state having slightly lower energy barrier $(0.582 \mathrm{eV}$ instead of $0.610 \mathrm{eV}$ in path 7$)$. From the $\mu_{2}$ transition state the $\mathrm{NH}_{2}$ can bind back to the surface on either side of the molecule, while it moves across the rows and/or switch footprint chirality (Fig. 3 (i)-(n)). The overall motion across the close-packed rows is accomplished by a repetition of the single steps in paths 5 to 7 (or 8) and then path 7 (or 8) to 1 in succession, Fig. 3 (i) and (j). Fig. 3 lists the activation barrier in $[\mathrm{eV}]$ for the single step or the highest barrier for the multiple steps mechanisms (motion across the rows). Since for steps (g) to (l), multiple paths are available, we report 
the barriers for each of them. From these paths the rate limiting steps are associated with the highest energy barrier between minimum and transition state, which we therefore chose to consider in the coarse grained kinetic model. The 14 pathways summarised in Fig. 3 serve as input for our stochastic model. The summary focuses on the state to state dynamics of a molecule from one energy minimum to the next, accounting indirectly for the $\mu_{2}$ transition state geometries through the energy barriers.

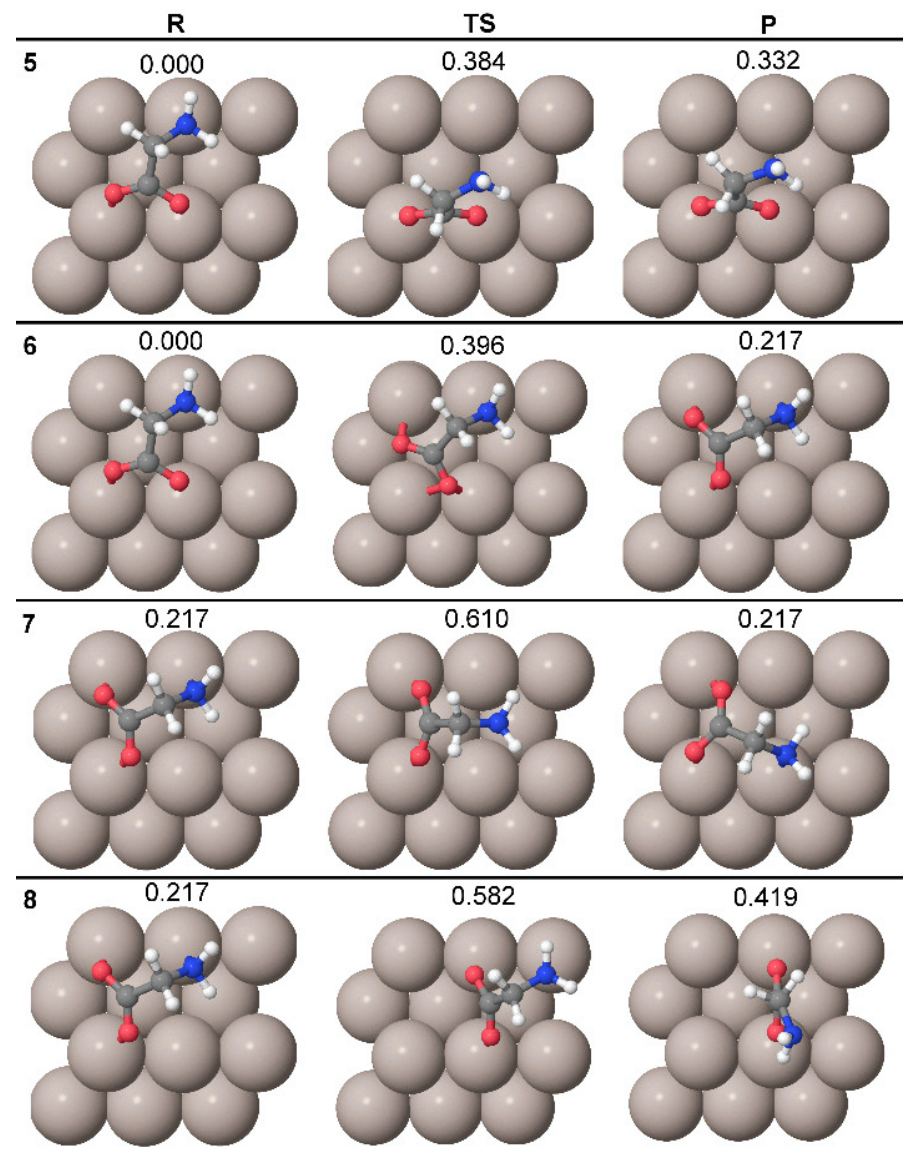

Figure 6: Diffusion across the close-packed $\mathrm{Cu}\{110\}$ rows: ${ }^{92}$ paths 5 to 8 , see numbers on the left, with initial geometry ( $\mathrm{R}$ or reactant) on the left panel, the first transition state structure (TS) of the path in the centre, and the first intermediate minimum as product (P) on the right. Above each configuration the energy difference is given in $\mathrm{eV}$.

The DFT energy barrier heights in Fig. 5 and Fig. 6 are compatible with facile surface phenomena at room temperature. The combination of motions along and across the closepacked rows, and the ability to switch footprint chirality, allow the adsorbed monomers to join together in islands formed by interlinked, hydrogen-bonded, heterochiral domains 
(Fig. 2). ${ }^{29}$ The expected outcome of diffusion and self-assembly is formation of islands and terraces of glycine bound by hydrogen-bonds in $\mathrm{p}(3 \times 2)$ pg heterochiral domains with glideline symmetry. In Fig. 2, the green-red island (b) and the yellow-blue island (a) are identical in terms of overall (racemic) chirality (and energy), but are oriented in the opposite direction with respect to the substrate, as observed in experiments. ${ }^{29}$ The pattern shows up as a zigzag distortion in an LEED image, similar to results for ALA. ${ }^{112}$

\section{Results and Discussion}

\section{The Structure of Homochiral Arrangements}

The homochiral arrangements we expect, Fig. 2 (c)-(d), are distinct from what has been suggested in the literature so far. Their periodic vector is $\{(3,0),(2,1)\}$. While the heterochiral $\mathrm{p}(3 \times 2) \mathrm{pg}$ arrangement has been confirmed by several experimental studies the models proposed so far for the homochiral pesudo-centered $(3 \times 2)$ phase are inconsistent with at least one experimental study. ${ }^{113}$ Heterochirality is not enough to explain the near extinction of missing spots in the LEED patterns and other experiments. ${ }^{17,20,29,113}$ We therefore expect a (near) glide plane symmetry in homochiral clusters. Some of the studies mention that artefacts are possible due to the asymmetry of the tunnelling tip in STM or displaced copper atoms. Nonetheless the guesses for the approximate $\mathrm{c}(3 \times 2)$ arrangements of homochiral clusters include some rotated molecules, Fig. 7 (a)-(d). The first, in Fig. 7 (a) where half of the glycinate species have undergone a small azimuthal rotation is a suggestion based on DFT calculations. ${ }^{23,29,93,113}$ Apart from the structure being close to a metastable intermediate minimum of the pathways mentioned before, the glide plane symmetry is missing and the offsite oxygens were invisible in photoelectron diffraction experiments. ${ }^{113}$ Neither leads displacing molecules by the vector $\frac{2}{3} \overrightarrow{a_{1}}+a_{2}$ as suggested by STM measurements, Fig. 7 (b), to a glide plane symmetry. ${ }^{20}$ Fig. 7 (c) shows an arrangement that is close to the $\mathrm{c}(3 \times 2)$ observed in STM, but inconsistent with photo-diffraction measurements, with glycinate molecules off- 
set to local sites such that the carboxylate species are midway between bridging and atop sites. ${ }^{20,113}$ The glide-plane symmetry is only violated by the relative positions of the amino groups and the $\mathrm{H}$ atoms bonded to the $\mathrm{C}^{*}$ chiral centre. For the fourth guess, Fig. 7 (d) half of the glycinate adsorbates have been rotated by $180 \mathrm{deg}$ relative to the surface normal and translated such that no surface $\mathrm{Cu}$ atoms has more than one $\mathrm{O}$ or $\mathrm{N}$ near neighbour. Here the desired symmetry would come from the dimers together. However, the adjacent O atoms lie to close. ${ }^{113}$

We find that a periodic vector $\{(3,0),(2,1)\}$ produces a homochiral arrangement that matches all of the experimental findings. First of all it has a glide plane symmetry. Then the arrangement explains why the $\mathrm{CH}-\mathrm{O}$ hydrogen bonds along a diagonal, as found in STM measurements,${ }^{20}$ are stronger in the homochiral clusters, see Fig. 7 (e)-(f). They are, however, unlike the previously suggested $(3 \times 2)$ diagonal, along the diagonal of the primitive $\mathrm{Cu}\{110\}$ cell.

To understand how our arrangement is consistent with a pseudo-centric $(3 \times 2)$ cell we start from the green $(3 \times 2)$ cell in Fig. 7 (e) that matches the heterochiral arrangement, as suggested in Fig. $6 \mathrm{~b}$ of Chen et. al. ${ }^{20}$ If the corner sites of the cell are on-top sites then the center is a bridge site and vice versa. Experimental studies concluded that when choosing to put the corner sites midway between on-top and bridge sites then the center sites are equivalent. However, the blue $(3 \times 2)$ unit cell in Fig. 7 (e) lacks resemblance with the experimentally observed symmetry. The slight displacement of the centre molecule in the STM results has so far been attributed to a potential artefact in the measurements, a defect on the copper surface, and slight rotations or shifts in the adsorbed GLY molecules, Fig. 7 (a)-(b). Instead we now take a $(3 \times 2)$ cell and skew it a bit, such that it becomes $\{(3,0)$, $(2,-1)\}$ periodic, see green cell in Fig. 7 (f). Shifting the corner of the skewed cell between on-top and bridge sites brings one molecule exactly into its centre. The resulting unit cell, blue in Fig. 7 (f), contains two amino groups at the bottom corners (tips of red triangles) and two groups with oxygen atoms at the top corners. Such a cell matches exactly the 
(a)

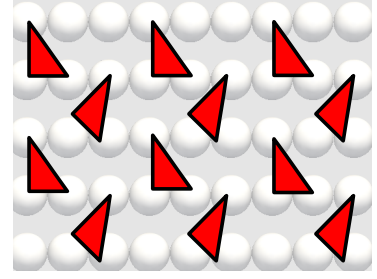

(c)

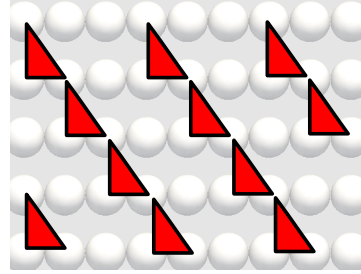

(e)

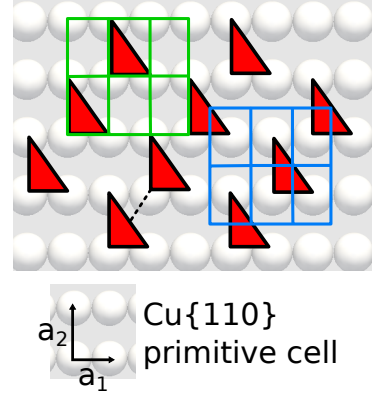

(b)

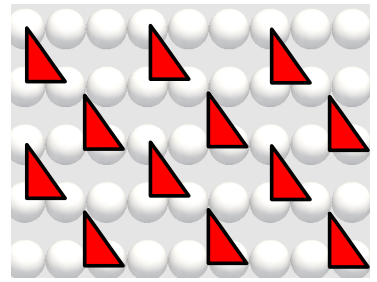

(d)

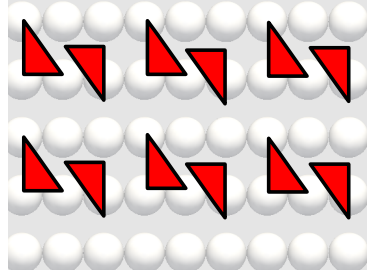

(f)

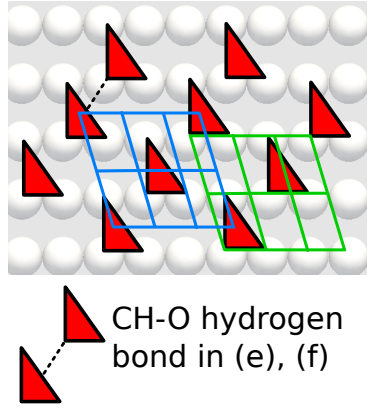

Figure 7: Comparison of homochiral clusters with previous guesses of pesudo-centered $(3 \times 2)$ arrangements: (a) slightly rotated centre molecule, ${ }^{23,29,93,113}$ (b) by $\frac{2}{3} \overrightarrow{a_{1}}+a_{2}$ displaced molecules, ${ }^{20,29}$ (c) offset to local sites, ${ }^{113}$ (d) half of the molecules rotated, ${ }^{113}$ (e) and (f) are glide plane symmetric clusters, where shearing and translating the unit cell so that its corners lie between bridge and atop sites reproduces the experimentally ${ }^{20}$ observed pesudo-centered $(3 \times 2)$ phase, blue in $(\mathrm{f})$.

arrangement in Fig. $6 \mathrm{~b}$ of Chen et. al. ${ }^{20}$ and explains the measurements that predicted near $c(3 \times 2)$ glide plane symmetry. ${ }^{113}$ Therefore, we argue that in the case of homochiral clusters a periodicity of $\{(3,0),(2,1)\}$, as shown in Fig. 2 (c) $-($ d), represents the pseudo-centric $(3 \times 2)$ phase observed in experiments.

\section{The Effects of Molecular Interaction Strength on Island Formation}

We first investigated the role of the non-covalent interaction strength using four different additional barriers $\Delta E_{H}$ of $0.075 \mathrm{eV}, 0.1 \mathrm{eV}, 0.125 \mathrm{eV}$, and $0.15 \mathrm{eV}$ in the rate calculations. We 
compared the results at $350 \mathrm{~K}, 400 \mathrm{~K}, 450 \mathrm{~K}$, and $500 \mathrm{~K}$. With a barrier of $0.1 \mathrm{eV}$ the molecules were mobile enough to form larger, uniform homo- and heterochiral clusters without vacancies on the microsecond timescale (SI Fig. S28-S61), as observed in experiments. ${ }^{29}$ In contrast, at $0.125 \mathrm{eV}$ and $0.15 \mathrm{eV}$ the clusters were smaller and were distributed more widely over the metal surface. The clusters remained separate with a barrier of $0.15 \mathrm{eV}$ at $400 \mathrm{~K}$ even after $3.057 \mu \mathrm{s}$, and still maintained their different anisotropies at $500 \mathrm{~K}$ after $1.998 \mu \mathrm{s}$. A barrier of $0.075 \mathrm{eV}$ resulted in weak association between the molecules. The heterochiral clusters disintegrated and homochiral clusters with high mobility were favoured. For a barrier of $\Delta E_{H}=0.1 \mathrm{eV}$ all the experimentally observed behaviour was qualitatively reproduced: the homochiral clusters were less stable than the heterochiral clusters, and the time scales to form the first enantiopure clusters lay between $0.015 \mu \mathrm{s}(450 \mathrm{~K})$ and $0.137 \mu \mathrm{s}(400 \mathrm{~K})$. We therefore chose $\Delta E_{H}=0.1 \mathrm{eV}$ for all further simulations. Since the non-covalent bond strength is $2 \Delta E_{H}=0.2 \mathrm{eV}$ by detailed balance, i.e. the ratio of the forward and reverse rates to form or break a bond, the chosen value aligns well with the previously estimated $0.22 \mathrm{eV} .{ }^{100}$

Our comparisons of different values for the molecular interaction strength confirm their crucial role in island formation. Pattern formation in the GLY islands results from a complex balance of hydrogen bonding, van der Waals forces, and dipole-dipole interactions.

\section{The Mechanism of Island Formation}

Time resolved dynamics of cluster and island formation of GLY molecules on $\mathrm{Cu}\{110\}$ is at the present an open research question from an experimental point of view. Fig. 8 displays snapshots at $400 \mathrm{~K}$ over the simulation time. Periodicity is indicated in all our images by duplicated atom rows on the right and top of the $20 \times 20$ cluster with slightly darker colour. Initially, 50 GLY molecules are randomly distributed on the $\mathrm{Cu}\{110\}$ surface. After $0.137 \mu \mathrm{s}$ the first homochiral cluster (yellow) forms, and increases in size until $0.332 \mu \mathrm{s}$. A second smaller homochiral cluster (red) appears after $0.509 \mu \mathrm{s}$. At $0.676 \mu \mathrm{s}$ two heterochiral clusters with different anisotropies are visible (blue-yellow and red-green). These clusters melt by 
turning back into homochiral configurations, either blue or yellow [Fig. 8 (g)-(i)]. Formation of the final heterochiral cluster takes at around $1.949 \mu \mathrm{s}$. Then the cluster remains stable for several microseconds [Fig. $8(\mathrm{k})]$.

We draw the following conclusion about the mechanism of island formation: to melt and merge into a larger cluster, smaller heterochiral clusters need to become slightly unstable and turn into mainly homochiral clusters before they can melt and then be heterochiral again. The observed initial assembly of smaller clusters from randomly distributed molecules in homochiral clusters with intermediate stability supports this picture. The advantage of first forming a less stable enantiopure cluster is that molecules can associate and dissociate more easily from it, retaining surface mobility until larger assemblies form.

The same mechanism was observed for the melting of two enantiopure clusters (18 molecules, 0.135 ML coverage) (Fig. 9). To avoid a conformational bias we added homochiral clusters to the surface that were slightly different from the expected cluster with periodic vectors of $\{(3,0),(2,1)\}$. Within $0.023 \mu \mathrm{s}$ the clusters align diagonally. At $0.073 \mu \mathrm{s}$ we observe homochiral cluster melting with periodicity as expected. Then the cluster splits into two groups of the same anisotropy (blue-yellow). Further evidence for the suggested mechanism is provided in SI Fig. S63.

\section{The Influence of Chirality in the Initial Configuration}

For a heterochiral, racemic island of 20 molecules with a neighbouring heterochiral island of four molecules, the islands melt within $1.365 \mu \mathrm{s}$. Single abstractions from the smaller island occur at $500 \mathrm{~K}$ after $0.164 \mu \mathrm{s}, 0.330 \mu \mathrm{s}$, and $0.764 \mu \mathrm{s}$ (SI Fig. S72 and the related movie). However, instead of associating into the cluster, the single molecule remaining at the end drifts further away from the large island to locate the optimal distance from both sides, facilitated by the periodic boundary conditions. If the bigger island consists of 16 molecules a single additional molecule on the surface integrates into the cluster within $0.503 \mu \mathrm{s}$. In contrast, when the larger heterochiral island comprises 16 molecules and the 


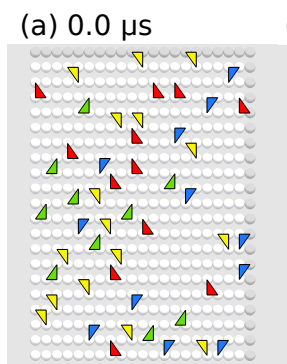

(b) $0.137 \mu \mathrm{s}$

(c) $0.177 \mu \mathrm{s}$

(d) $0.332 \mu \mathrm{s}$
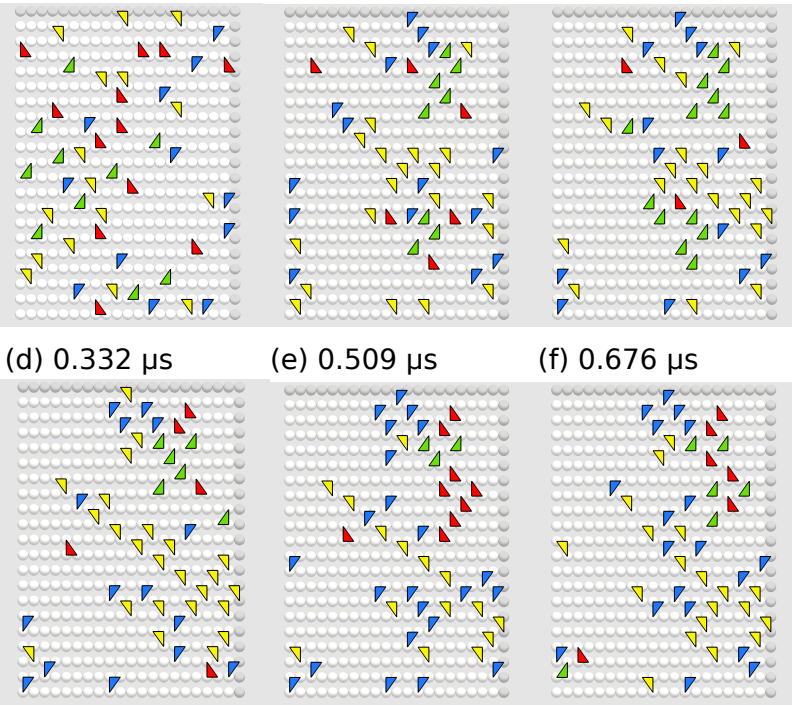

(e) $0.509 \mu \mathrm{s}$

(f) $0.676 \mu \mathrm{s}$
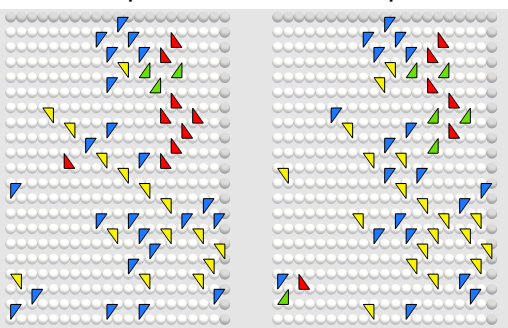

(g) $1.186 \mu \mathrm{s}$

(h) $1.352 \mu \mathrm{s}$

(i) $1.703 \mu \mathrm{s}$
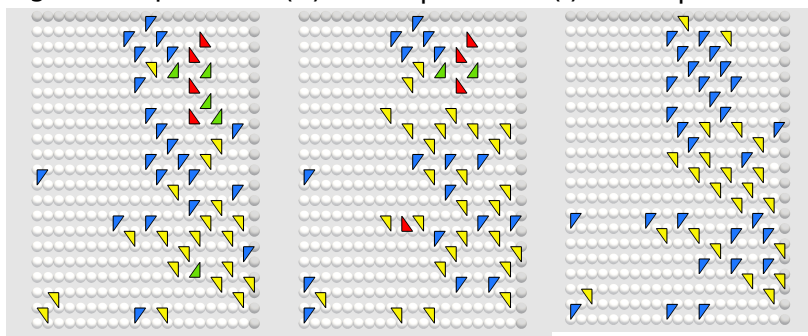

(j) $1.949 \mu \mathrm{s}$

(k) $6.053 \mu \mathrm{s}$
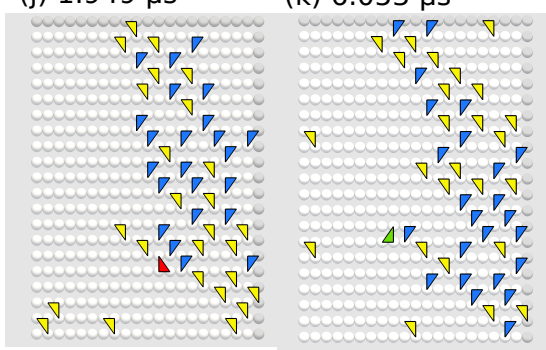

Figure 8: The molecular kinetics of 50 randomly initialised GLY molecules at $400 \mathrm{~K}$. The duplicated atom rows on the top and right in slightly darker colours indicate periodic boundary conditions.

smaller 6 (0.2175 ML coverage), the islands do not change chirality or melt within $1.405 \mu \mathrm{s}$ over a distance of three copper top layer rows. As expected, smaller islands are less stable than bigger clusters. Clearly, the size of the island determines its stability, and a network of six heterochiral molecules is bound more tightly than an island of four.

Islands in contact with a perfect racemic mixture of different anisotropies and nine molecules each reorganise into the same anisotropic direction, Fig 10. Two heterochiral 


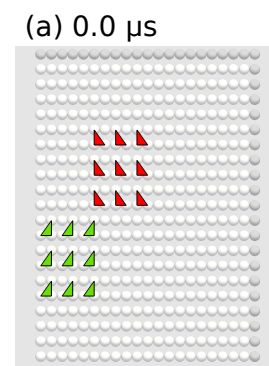

(d) $0.192 \mu \mathrm{s}$

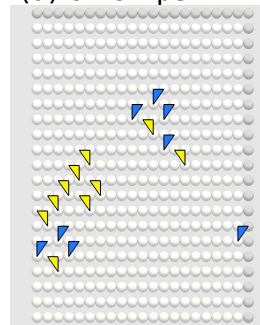

(b) $0.023 \mu \mathrm{s}$

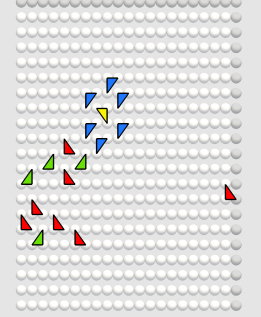

(e) $2.230 \mu \mathrm{s}$

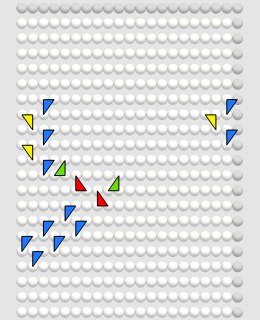

(c) $0.073 \mu \mathrm{s}$

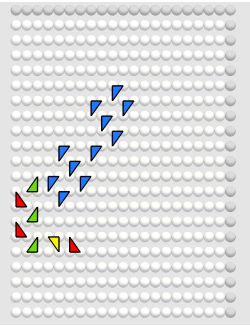

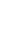

Figure 9: Cluster melting of two homochiral clusters at $500 \mathrm{~K}$.

(a)

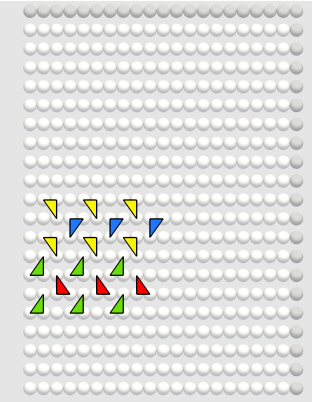

(c)

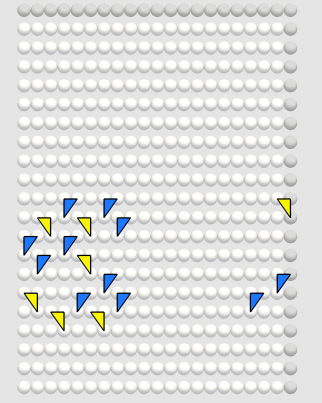

(b)

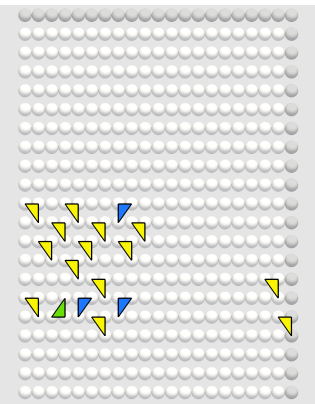

(d)

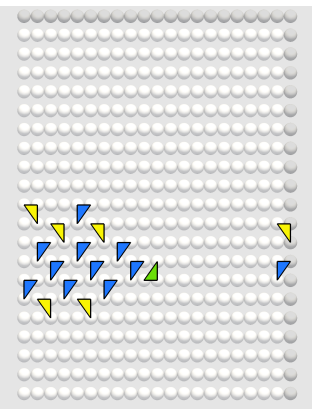

Figure 10: Reorganisation of heterochiral clusters in contact with different anisotropy (blueyellow and green-red) clusters at $500 \mathrm{~K}$ via the homochiral-heterochiral mechanism: after (a) $0.0 \mu \mathrm{s}$, (b) $0.046 \mu \mathrm{s}$, (c) $0.246 \mu \mathrm{s}$, and (d) $3.097 \mu \mathrm{s}$.

islands of ideal racemic mixture and with different molecular anisotropy and three copper top layer rows between them are too stable to merge together, even after $1.296 \mu \mathrm{s}$. When the initial configuration of the islands has the same anisotropy (Fig. 11) the three copper top 
layer rows between them are lowered to a shorter distance. The islands come into contact after $1.002 \mu \mathrm{s}$ and align with each other after $1.592 \mu \mathrm{s}$. If instead of three rows the islands are separated by only one row, they melt into a single island (SI Fig. S72). These tests show that the distance between clusters influences whether they start to melt or change their anisotropy. A greater separation reduces the attractive forces between two islands.

(a)

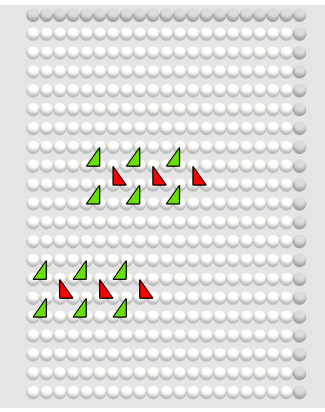

(c)

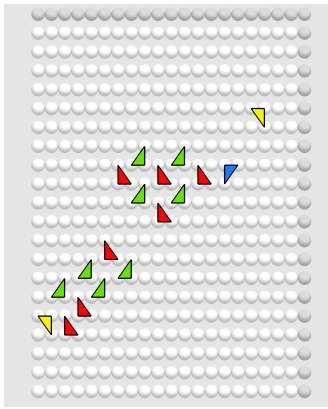

(b)

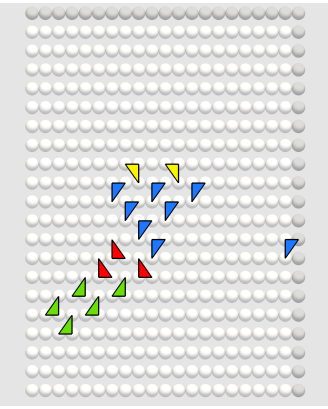

Figure 11: Two islands of the same anisotropy (green-red) at $500 \mathrm{~K}$ : snapshot of kinetics after (a) $0.0 \mu \mathrm{s}$, (b) $1.002 \mu \mathrm{s}$, (c) $1.592 \mu \mathrm{s}$.

When we start from an initial condition with two islands, an enantiopure and a heterochiral cluster with 50 molecules corresponding to $0.375 \mathrm{ML}$ coverage [Fig. 12 (a)], the heterochiral cluster is static, whereas the enantiopure, homochiral cluster melts into it. As for the mechanism described above, intermediate networks are established that include smaller heterochiral and homochiral clusters as connections. At $450 \mathrm{~K}$ (Fig. 13) the first molecules join to the larger racemic cluster after $0.873 \mu \mathrm{s}$. After $1.706 \mu \mathrm{s}$ the majority of molecules belong to one anisotropy (blue-yellow). In contrast, at $500 \mathrm{~K}$ the molecules nucleate into a second racemic cluster (red-green). The green homochiral cluster formed within $0.874 \mu \mathrm{s}$, turns into a stable heterochiral cluster. The more stable cluster, racemic and heterochiral at 
the start, incorporates a molecule from the initially enantiopure cluster. A temperature of $450 \mathrm{~K}$ is optimal, if the aim is to create a large heterochiral cluster of the same anisotropy, as confirmed by later examples. For both the $500 \mathrm{~K}$ and $450 \mathrm{~K}$ trajectories, movies are available as part of the SI.

(a)

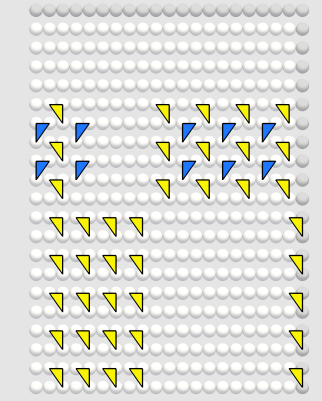

(c)

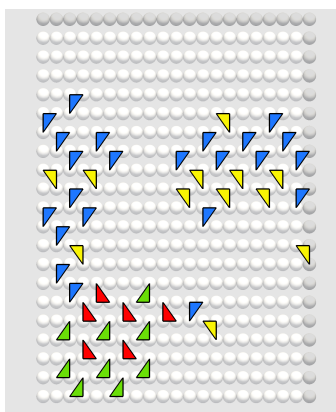

(b)

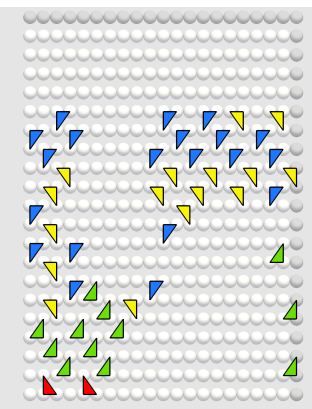

Figure 12: Melting of homochiral (yellow) and heterochiral (blue-yellow) clusters at $500 \mathrm{~K}$ : after (a) $0.0 \mu \mathrm{s}$, (b) $0.021 \mu \mathrm{s}$, (c) $0.949 \mu \mathrm{s}$.

At $400 \mathrm{~K}$ the clusters turn into one form of chirality (blue-yellow) within $6.052 \mu \mathrm{s}$, whereas at $350 \mathrm{~K}$ even after $6.906 \mu \mathrm{s}$ two heterochiral clusters of different anisotropy prevail. The reaction takes significantly longer due to the stability of the clusters at lower temperature.

We observe the same mechanism for 50 and 20 randomly initialised GLY molecules (0.375 and 0.15 monolayers coverage) (Fig. 14 and SI). At $400 \mathrm{~K}$ and $450 \mathrm{~K}$ the islands melt into one or two heterochiral clusters of the same direction (blue-yellow). For 50 molecules at $500 \mathrm{~K}$ and $350 \mathrm{~K}$ the islands formed keep two separate directionalities. A low coverage $(0.15$ monolayers) the islands are too small to assign anisotropy, apart from the bottom left cluster at $350 \mathrm{~K}$ displaying heterochirality (green-red) after $12.841 \mu \mathrm{s}$, which is an unlikely event 
(a)

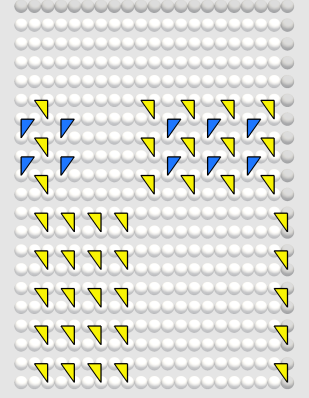

(c)

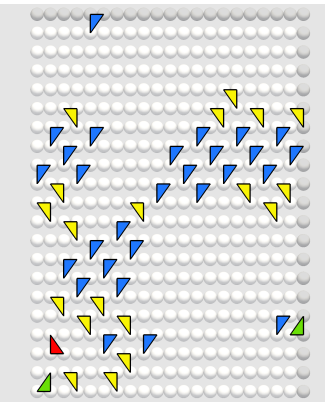

(b)

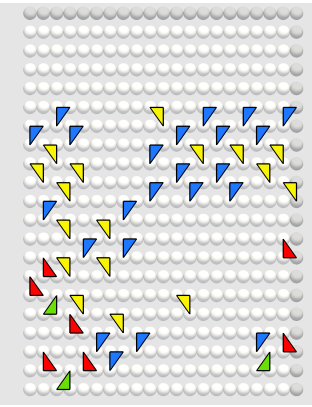

Figure 13: Melting of homochiral (yellow) and heterochiral (blue-yellow) clusters at $450 \mathrm{~K}$ : after (a) $0.0 \mu \mathrm{s}$, (b) $0.874 \mu \mathrm{s}$, (c) $1.706 \mu \mathrm{s}$.

due to the length of time required to reach this state.

Generally, the optimal melting and ordering temperature depends on which outcome is preferred, either a heterochiral mixture with islands of two different orientations, or one unified racemic island consisting of molecules with the same anisotropy. Experimentally, ${ }^{17,21}$ a temperature of $420 \mathrm{~K}$ is used to anneal the molecules, and a value above $408 \mathrm{~K}$ was found to be necessary. The surface requires annealing at about $420 \mathrm{~K}$ to produce an ordered $\mathrm{p}(3 \times 2)$ pg adlayer. ${ }^{17}$ In our simulations the predicted temperature range is influenced by potential variations in the DFT energies, which serve as input for the kinetic model. Therefore, the qualitative behaviour of the molecules can be captured between $400 \mathrm{~K}$ and $500 \mathrm{~K}$. The behaviour changes at higher coverage.

\section{Coverage Dependence}

The final states at three of the temperatures we tested for 100 GLY molecules (0.75 monolayers) consist of a heterochiral cluster with molecules of the same green and red anisotropy 
(a)

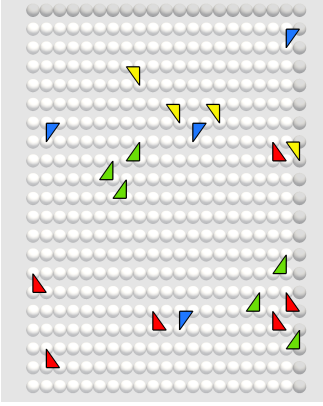

(c)

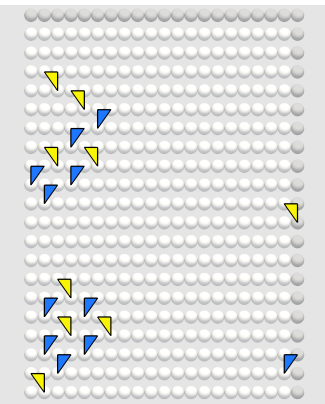

(b)

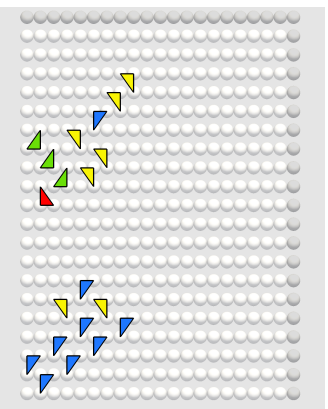

Figure 14: Molecular kinetics of 20 GLY molecules on $\mathrm{Cu}\{110\}$ at $400 \mathrm{~K}$ after (a) $0.0 \mu \mathrm{s}$, (b) $0.020 \mu \mathrm{s}$, (c) $1.745 \mu \mathrm{s}$

$(500 \mathrm{~K}, 450 \mathrm{~K}, 400 \mathrm{~K})$, while the fourth case $(350 \mathrm{~K})$ has a majority of the blue and yellow triangle anisotropy after $5.332 \mu \mathrm{s}$. We conclude that at higher coverage there is a stronger driving force for molecules to merge into connected heterochiral clusters of the same anisotropy. Attractive forces pulling the non-conforming molecules into larger heterochiral clusters seem to be stronger than at medium or low coverage.

At medium coverage ( 0.375 monolayers) a uniformly connected cluster is formed at $400 \mathrm{~K}$ (Fig. 8), whereas a few smaller clusters are established at $500 \mathrm{~K}$ and $450 \mathrm{~K}$. At $350 \mathrm{~K}$ the molecules are slowly moving, if they move at all, and, thus, despite the larger clusters attracting single molecules, there are several smaller assemblies on the copper surface (SI Sections 1). Similarly, the 100 molecules at $0.75 \mathrm{ML}$ coverage exhibit little or no compacting behaviour at $350 \mathrm{~K}$. To visualise this effect we calculated the COM of each molecule represented by a triangle and plotted the distribution of Euclidean distances between the COMs over time. The COM analysis of 100 self-assembling molecules leads to three clear dark red peaks in Fig. 15 (top) after $1.776 \mu \mathrm{s}$. The corresponding dynamics on the surface, which follows the 
same mechanism in homochiral and then heterochiral cluster formation as described above, is shown in Fig. 16. After $1.776 \mu \mathrm{s}$ [Fig. $16(\mathrm{~d})]$ the many homochiral parts of the cluster indicate that it will undergo further compaction. The COM analysis of 100 molecules at $350 \mathrm{~K}$ shows only the shortest distance peak of the three at $450 \mathrm{~K}$, indicating that the molecules have several next-nearest neighbours, but fewer second- and third-nearest neighbours, as expected in a less dense cluster. At medium coverage (0.375 monolayers, $450 \mathrm{~K})$ (Fig. 15 (middle) and SI Fig. S7) the molecules first build smaller clusters at a similar distance. Over time the clusters grow, and the lowest COM distance count increases as well as those for longer distances, because the separation between the clusters simultaneously increases. A temperature of $450 \mathrm{~K}$ is advantageous for assembly of larger connected clusters at medium coverage.

The peak accumulation of COM distances at low coverage (0.15 ML, $450 \mathrm{~K}$ ) (Fig. 15 (bottom) and SI Fig. S28) originates from the three clusters that are formed within $3.517 \mu \mathrm{s}$. In comparison, at $350 \mathrm{~K}$ the six initial clusters melt into four by incorporating single molecules within $12.841 \mu \mathrm{s}$. At $400 \mathrm{~K}$ (Fig. 14) the 20 molecules melt into two clusters within $0.020 \mu \mathrm{s}$.

At $500 \mathrm{~K}$ the molecules are highly mobile and avoid larger aggregation, as expected when entropy has a more significant effect. They start aligning along the diagonal within $0.019 \mu \mathrm{s}$. After $0.106 \mu \mathrm{s}$ small clusters have formed. The first homochiral cluster containing six molecules (red) appears after $0.259 \mu \mathrm{s}$. In the end $(1.687 \mu \mathrm{s})$ three clusters remain (Fig. 17). In summary, at low coverage a temperature between $400 \mathrm{~K}$ and $450 \mathrm{~K}$ is again optimal if we are interested in larger aggregations of molecules.

\section{The Role of Defects}

For medium and low coverage we looked at the role of defects on the metal surface. Defects were represented by areas on the $\mathrm{Cu}\{110\}$ surface where GLY was excluded. These areas mimic defects induced through ad-atoms in alloys. For example silver ad-atoms could block the moves of the adsorbed GLY molecules due to their large bridge site. We found that 


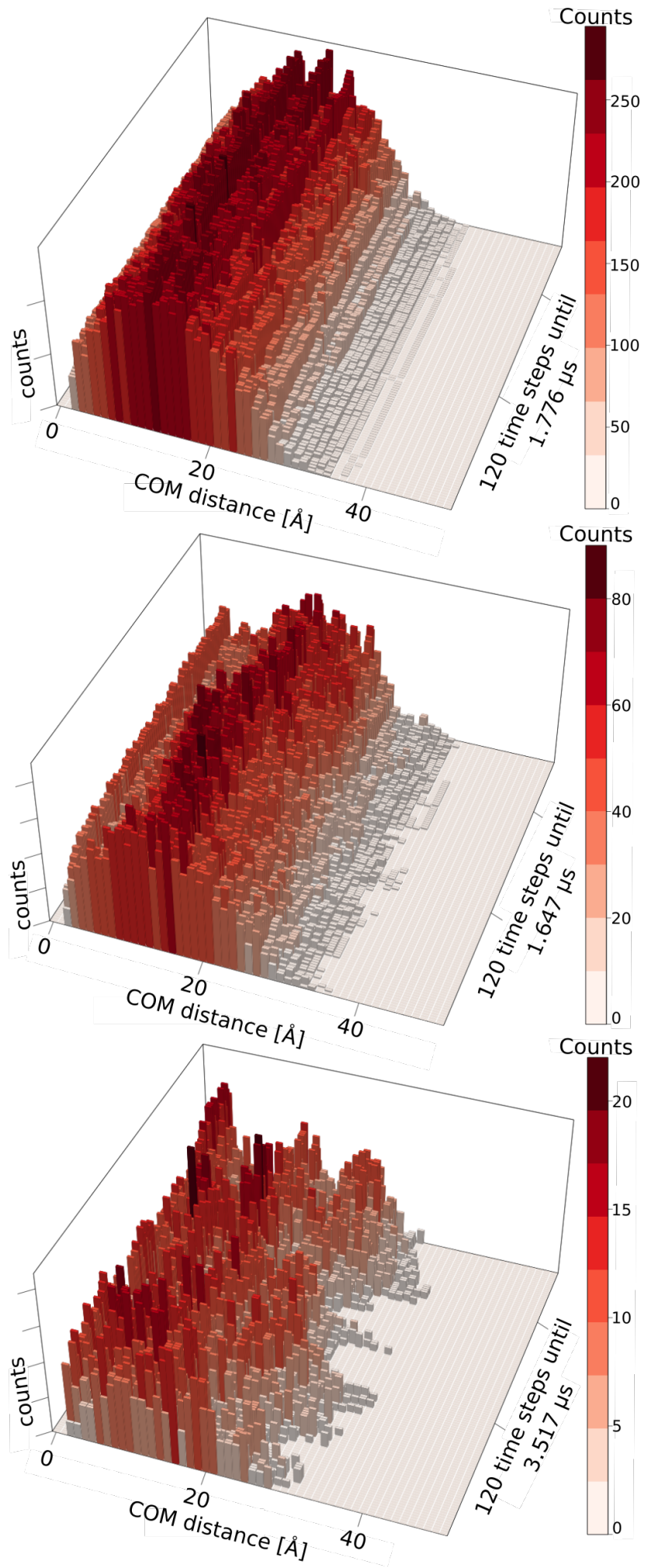

Figure 15: Centre of mass distance evolution over time at $450 \mathrm{~K}$, molecular interaction strength $\Delta E_{H}=0.1 \mathrm{eV}$ for 100 molecules (top), 50 molecules (middle) 20 molecules (bottom). 
(a)

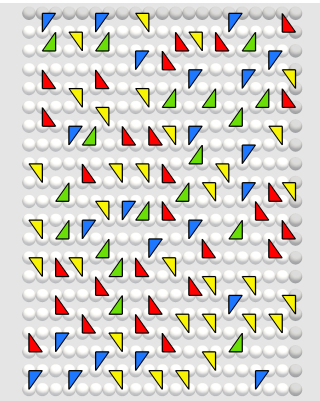

(c)

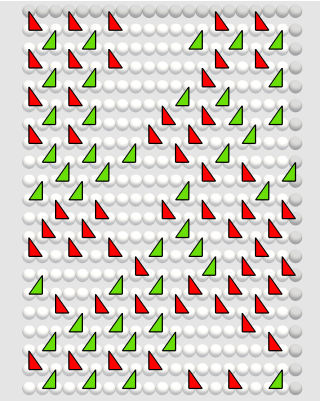

(b)

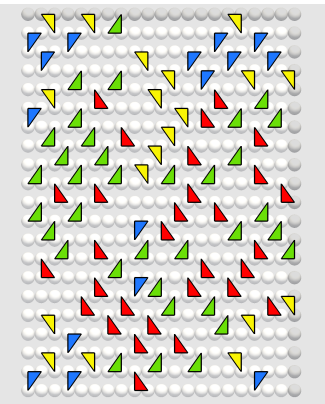

(d)

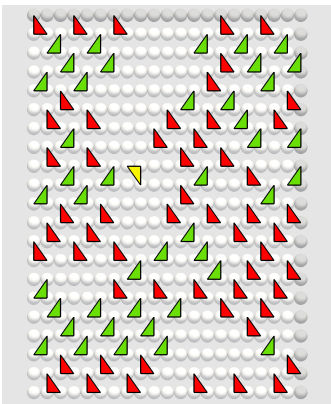

Figure 16: Self-assembly of 100 molecules at $450 \mathrm{~K}$ : after (a) $0.0 \mu \mathrm{s}$, (b) $0.020 \mu \mathrm{s}$, (c) $1.745 \mu \mathrm{s}$, (d) $1.776 \mu \mathrm{s}$.

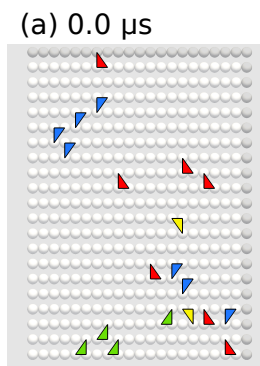

(d) $0.259 \mu \mathrm{s}$

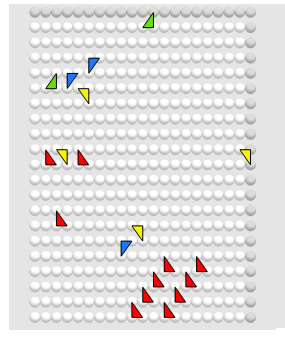

(b) $0.019 \mu \mathrm{s}$

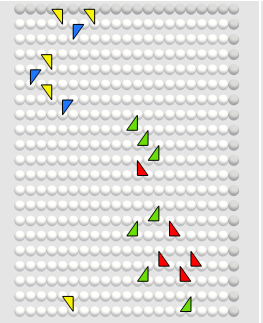

(e) $1.687 \mu \mathrm{s}$

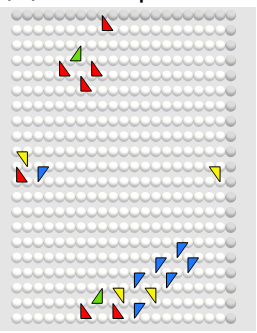

(c) $0.106 \mu \mathrm{s}$

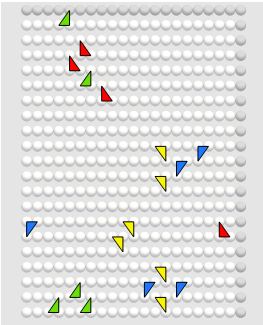

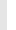


into heterochiral clusters at $500 \mathrm{~K}$, despite the presence of defects. However, defects prevent the formation of a uniform anisotropy. The diagonal between the two defects marks the separation line for blue-yellow and green-red anisotropy, Fig. 18. At lower temperature, the role of the defects is, apart from keeping molecules with different anisotropy apart, the stabilisation of mainly homochiral final states. In terms of the mechanism described above: the molecules start to organise for potential assembly into a heterochiral cluster, but they never reach this point.

(a)

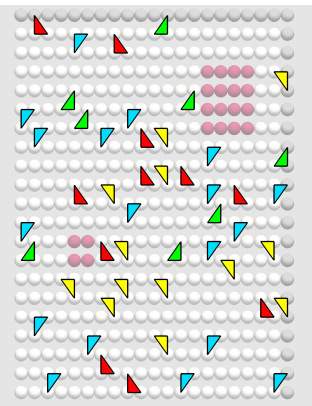

(b)

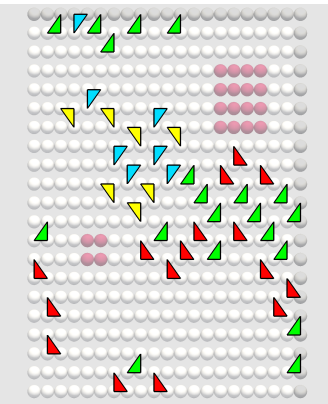

Figure 18: Molecular kinetics of 50 GLY molecules on $\mathrm{Cu}\{110\}$ with defects at $500 \mathrm{~K}$ after (a) $0.0 \mu \mathrm{s}$, (b) $0.761 \mu \mathrm{s}$.

\section{Comparison with Experiments}

We have successfully reproduced the essential features of the experimental results, such as the greater stability of heterochiral clusters compared to enantiopure. One explanation for this behaviour is that the racemic cluster can achieve denser packings in some cases. For example, a $4 \times 8 \mathrm{Cu}\{110\}$ surface without periodic boundary conditions can contain seven green GLY molecules and eight from a heterochiral green-red cluster. In both cases there are 8 hydrogen-bonds. Extending the copper surface slightly by one row provides the possibility of having the same number of molecules in both clusters, while maintaining the same number of hydrogen-bonds. The heterochiral cluster is therefore denser and more favourable compared to the homochiral clusters.

Our results indicate that the non-covalent interactions between GLY molecules are re- 
sponsible for pattern formation on the surface, in agreement with experimental observations on $\mathrm{Cu}\{531\}$, where the adsorption patterns of GLY molecules on the $\{110\}$ and $\{311\}$ sites is driven by hydrogen-bonds. ${ }^{114}$ This result can be compared with the thermal control of adenine chain rotations on $\mathrm{Cu}\{110\}$, where van der Waals interactions are critical. ${ }^{95}$

In agreement with LEED measurements, our results for stable clusters show a $\mathrm{p}(3 \times 2) \mathrm{pg}$ phase of GLY adsorbed on $\mathrm{Cu}\{110\} .{ }^{29}$ In our comparisons of different starting configurations we identified GLY islands consisting of a racemic mixture of molecules as the most stable. For instance, two of these islands remained separate, whereas the enantiopure islands merged together. Additionally, the racemic islands appear at the end of the island formation process. The melting occurs in enantiopure states. Our findings confirm previous calculations that a spontaneous segregation of molecular enantiomers does not occur in racemically adsorbed mixtures. $^{93}$

Investigations of ALA adsorbing on naturally chiral $\mathrm{Cu}\{3,1,17\}^{\mathrm{R} \& \mathrm{~S}}$ showed that conglomerate phases are more energetically stable than a racemate phase, while the reported DFT simulations overestimate the enantiospecificity of the adsorption energetics. ${ }^{115}$ Racemic ALA adlayers on $\mathrm{Cu}\{110\}$ were found to be structurally analogous to those observed for GLY on this surface, and adopted a pseudo-racemate ordering. ${ }^{93}$ Low-energy electron diffraction (LEED), reflection absorption infrared spectroscopy (RAIRS), and STM studies of racemic and enantiopure ALA on $\mathrm{Cu}\{110\}$ found a similar preference for racemic clusters. ${ }^{112}$ An open question for experiments is therefore whether the same island formation mechanisms can be observed for ALA on $\mathrm{Cu}\{110\}$ as for GLY. Moreover the binding configuration of GLY on $\mathrm{Cu}\{110\}$ is almost identical to that of ALA $\mu_{3}$, the energy barriers that were calculated for GLY suggest a similarly flat energy landscape for ALA on the $\mathrm{Cu}\{110\}$ surface.

We expect to find similar behaviour in serine compared to ALA and GLY. However, racemic mixtures of serine on the $\mathrm{Cu}\{110\}$ surface prefer to order into enantiopure islands by forming homochiral subunits and establishing directional hydrogen-bonds, ${ }^{96}$ whereas GLY is more stable in the heterochiral state. In contrast to serine, GLY molecules exhibit only two 
levels of chirality in the enantiopure state, which may cause the different preferences, because serine expresses three levels of chirality in enantiopure clusters in its anionic state: (a) dimer orientation with respect to the substrate, (b) chiral substructure lattices for aggregates of 1 and d-enantiomer dimers, and (c) chirality of the molecules influence the form of elongated shapes of small islands. Furthermore, the packing of the molecules can be denser, depending on the size of the surface, for heterochiral, racemic GLY clusters. A denser packing means that the interacting forces can hold clusters together more strongly.

For ALA on $\mathrm{Cu}\{110\}$ racemic systems appear with a $(3 \times 2)$ phase in experiments at high coverage. Our findings for heterochiral GLY clusters agree with these results. We identified a different periodicity for the enantiopure cluster than the ALA $(3 \times 2)$ phase. Unit cells with a periodic vector of $\{(3,0),(2,1)\}$ reproduce the pseudo-centered $(3 \times 2)$ phase observed in experiments with GLY. While the enantiopure phase is only achieved at temperatures more than $100 \mathrm{~K}$ higher than required for the racemic ALA $(3 \times 2)$ phase, we identified a different mechanism for GLY. The enantiopure phase appears as an important intermediate for the GLY islands in the process of island melting. The homochiral clusters have stronger CH-O hydrogen bonds along the diagonal of the $\mathrm{Cu}\{110\}$ primitive cells. ${ }^{20}$ These linear diagonal forces can counteract the NH-O hydrogen bonds along the [011] rows that seem to be mainly responsible for the network effects in heterochiral clusters. Thus, the heterochiral clusters are more stable and hold stronger together. The enantiopure phase was classified as a small minority phase on the surface. ${ }^{113}$ Its estimated coexistence with heterochiral clusters is about $15 \%$ of the coverage. ${ }^{29}$ Most of these experiments use techniques where probes are taken after cluster formation has finished and the less stable homochiral clusters have turned into racemic clusters. Therefore, an earlier probing may result in a proportion higher than $15 \%$ for the enantiopure phase.

If we add defects to the surface at medium coverage, the enantiopure phase is preferred between $400 \mathrm{~K}$ and $450 \mathrm{~K}$. In this case the defects could induce a seed effect that strengthens the diagonal $\mathrm{CO}-\mathrm{H}$ interactions and blocks the rearrangement into a cross-diagonal network 
where the NH-O are of greater importance. From the comparisons of hydrogen-bond strength effects, the final states are enantiopure at $450 \mathrm{~K}$ and $500 \mathrm{~K}$ for a weaker hydrogen-bond with lower barriers $(0.75 \mathrm{eV}$ instead of $0.1 \mathrm{eV})$. On the other hand, 100 GLY molecules at high coverage reach a mainly enantiopure state after $1.776 \mu \mathrm{s}$ at $450 \mathrm{~K}$ and $\Delta E_{H}=0.1 \mathrm{eV}$ (Fig. 16). The similarity in behaviour of high coverage, defect free surfaces, and medium coverage defective surfaces can be explained by considering the addition of a defect as an increase of surface coverage. The formation of similar patterns for a weaker $\Delta E_{H}$ barrier and medium coverage may be due to the fact that at higher coverage the complex interactions between the GLY molecules are balancing out forces, since most of the molecules have two direct neighbours pushing from the sides. Therefore the weaker attractive forces between the molecules at $\Delta E_{H}=0.75 \mathrm{eV}$ and medium coverage can lead to a similar result. For high coverage our simulations also agree with experimental results for the periodicity of the racemic arrangements: ${ }^{112}$ in neither case are the structures fully periodic over distances greater than a few molecules.

Since our rates are based on DFT energies, along with the assumptions intrinsic to Eyring's rate theory, the assumption that we can consider the motions of the molecules as independent Poisson processes, and an empirically fitted molecular interaction strength, our predictions should be regarded as qualitative. The uncertainties in the DFT energy based rates have a significant effect on the timescales and the temperature ranges we predict. Another reason to be cautious about rates is the local model, where a single molecule moves over each time step. Moreover, the approximate description of thermodynamics and the estimated treatment of the effects that molecular interactions have on the hopping rates leave flexiblity to design an even more realistic model. We have showed that our results are in qualitative agreement with experimental observations. To reach quantitative agreement, lengthy implementations of models with improved description of thermodynamics as well as uncertainty and sensitivity estimation techniques, available for methods similar to our algorithm, ${ }^{116,117}$ would be required. 
For future experiments with GLY on $\mathrm{Cu}\{100\}$ open questions include whether the different behaviour in terms of anisotropy alignment can be reproduced (a) at low and high coverage and (b) for various starting distributions of the molecules both with and without defects on the $\mathrm{Cu}\{110\}$ surface. For (S)-proline on $\mathrm{Cu}\{100\}$ it was possible to engineer homochiral footedness by a small structural modification. ${ }^{118}$ Such tuning of self-organisation of the molecules, as well as their outward facing functionalities, might be achieved in GLY clusters using specifically designed defects on the surface, if, for example, the homochiral cluster is preferred.

\section{Summary and Conclusion}

We have investigated the chemical kinetics and diffusion of GLY molecules on $\mathrm{Cu}\{110\}$. The GLY molecules move via seven possible diffusion pathways, identified with DFT calculations, from one conformer to the next, while changing footprint chirality. From these pathways we constructed a stochastic discrete event algorithm, an extension of KMC with $\tau$-leaping to study the formation of GLY clusters, networks, and islands. Our results reproduce the experimentally observed formation of enantiopure and racemic networks via the self-assembly of anisotropic GLY molecules into clusters. We confirm that a GLY arrangement with glide plane symmetry is possible for homochiral clusters. Unit cells with a periodic vector of $\{(3,0),(2,1)\}$ reproduce the experimentally observed pseudo-centered $(3 \times 2)$ phase. The mechanism of island formation first involves the establishment of intermediate homochiral clusters, then more stable heterochiral islands are formed. When two islands merge they first transform into the same enantiopure cluster before establishing a larger racemic network. The timescale of island melting and formation lies in the nanosecond range. The optimal hydrogen-bond strength in our model, leading to the formation of stable racemic clusters, is $0.1 \mathrm{eV}$. As expected, smaller islands were found to be less stable than larger clusters. We also consider the melting of homo- and heterochiral islands. A temperature between $400 \mathrm{~K}$ 
and $450 \mathrm{~K}$ is predicted to be optimal if the aim is to create a large racemic cluster of the same anisotropy. The optimal melting and ordering temperature depends on the preferred outcome, either a racemic mixture with several islands of two different orientations, or one unified racemic island containing molecules of the same anisotropy. At higher coverage the molecules experience a greater driving force to move into connected heterochiral clusters of the same anisotropy than at medium coverage. At medium coverage the defects prevent anisotropically oriented homogenisation of heterochiral clusters and stabilise mainly homochiral final states at lower temperature. Our results provide a connection between the observations from GLY kinetics and diffusion, experimental results, and possible mechanisms for several similar amino acids such as ALA, serine, and proline on the $\mathrm{Cu}\{110\}$ surface.

The insights and mechanisms discussed, especially the control of self-organisation via targeted defects, coverage, and temperature optimisation, bring us a step closer to tuning self-organisation of organic molecules on metal surfaces, as well as their functionality for SAMs in nano-fabrication.

\section{Acknowledgement}

J.B.R. thanks the Alexander von Humboldt foundation for a Fedor Lynen Research Fellowship and the British Engineering and Physical Sciences Research Council (EPSRC) for a Knowledge Transfer Fellowship.

\section{Supporting Information Available}

The code for the stochastic discrete event algorithm used in this study is available at Zenodo. ${ }^{102}$ The following files are available free of charge.

- supportingInfo.pdf: Overview of all results of the extended kinetics analysis.

- big34smaller12-new.mp4: melting of a smaller with a bigger island via single abstrac- 
tions (SI Fig. S72)

- 2islands500K-new.mp4: melting of a homochiral with a heterochiral cluster at $500 \mathrm{~K}$ (SI Fig. S14,S15,S59 ).

- 2islands450K-new.mp4: melting of a homochiral with a heterochiral cluster at $450 \mathrm{~K}$ (SI Fig. S12,S13,S55 ). 


\section{References}

(1) Gowers, S. A. N.; Curto, V. F.; Seneci, C. A.; Wang, C.; Anastasova, S.; Vadgama, P.; Yang, G.-Z.; Boutelle, M. G. 3D Printed Microfluidic Device with Integrated Biosensors for Online Analysis of Subcutaneous Human Microdialysate. Anal. Chem. 2015, 87, 7763-7770.

(2) Casalini, S.; Bortolotti, C. A.; Leonardi, F.; Biscarini, F. Self-assembled Monolayers in Organic Electronics. Chem. Soc. Rev. 2017, 46, 40-71.

(3) Langer, R.; Tirrell, D. A. Designing Materials for Biology and Medicine. Nature 2004, 428, 487-492.

(4) Gatto, E.; Venanzi, M. Self-assembled Monolayers Formed by Helical Peptide Building Blocks: a New Tool for Bioinspired Nanotechnology. Polym. J. 2013, 45, 468-480.

(5) Chaki, N. K.; Vijayamohanan, K. Self-assembled Monolayers as a Tunable Platform for Biosensor Applications. Biosens. Bioelectron. 2002, 17, 1-12.

(6) Loo, Y.; Zhang, S.; Hauser, C. A. From Short Peptides to Nanofibers to Macromolecular Assemblies in Biomedicine. Biotechnol. Adv. 2012, 30, 593-603.

(7) Fendler, J. H. Chemical Self-assembly for Electronic Applications. Chem. Mater. 2001, 13, 3196-3210.

(8) Badia, A.; Lennox, R. B.; Reven, L. A Dynamic View of Self-Assembled Monolayers. Acc. Chem. Res. 2000, 33, 475-481.

(9) Morrow, B. H.; Payne, G. F.; Shen, J. pH-Responsive Self-Assembly of Polysaccharide Through a Rugged Energy Landscape. J. Am. Chem. Soc. 2015, 137, 13024-13030.

(10) Diller, K.; Klappenberger, F.; Allegretti, F.; Papageorgiou, A. C.; Fischer, S.; Duncan, D. A.; Maurer, R. J.; Lloyd, J. A.; Oh, S. C.; Reuter, K. et al. Temperature- 
Dependent Templated Growth of Porphine Thin Films on the (111) Facets of Copper and Silver. J. Chem. Phys. 2014, 141, 144703.

(11) Yan, L.-L.; Tan, C.-H.; Zhang, G.-L.; Zhou, L.-P.; Bünzli, J.-C.; Sun, Q.-F. Stereocontrolled Self-Assembly and Self-Sorting of Luminescent Europium Tetrahedral Cages. J. Am. Chem. Soc. 2015, 137, 8550-8555.

(12) Vanderkooy, A.; Taylor, M. S. Solution-phase Self-assembly of Complementary Halogen Bonding Polymers. J. Am. Chem. Soc. 2015, 137, 5080-5086.

(13) Jacobs, W. M.; Reinhardt, A.; Frenkel, D. Rational Design of Self-assembly Pathways for Complex Multicomponent Structures. Proc. Natl. Acad. Sci. U. S. A. 2015, 112, $6313-6318$.

(14) Mark, A. G.; Forster, M.; Raval, R. Recognition and Ordering at Surfaces: The Importance of Handedness and Footedness. ChemPhysChem 2011, 12, 1474-1480.

(15) Forster, M.; Dyer, M.; Persson, M.; Raval, R. Assembly of Chiral Amino-Acids at Surfaces from a Single Molecule Perspective: Proline on $\mathrm{Cu}(110)$. Top . Catal. 2011, $54,13-19$.

(16) Barlow, S. M.; Raval, R. Nanoscale Insights in the Creation and Transfer of Chirality in Amino Acid Monolayers at Defined Metal Surfaces. Curr. Opin. Colloid Interface Sci. 2008, 13, 65-73.

(17) Barlow, S. M.; Raval, R. Complex Organic Molecules at Metal Surfaces: Bonding, Organisation and Chirality. Surf. Sci. Rep. 2003, 50, 201-341.

(18) Shavorskiy, A.; Aksoy, F.; Grass, M. E.; Liu, Z.; Bluhm, H.; Held, G. A Step Toward the Wet Surface Chemistry of Glycine and Alanine on $\mathrm{Cu}\{110\}$ : Destabilization and Decomposition in the Presence of Near-Ambient Water Vapor. J. Am. Chem. Soc. 2011, 133, 6659-6667. 
(19) Efstathiou, V.; Woodruff, D. P. Characterisation of the Interaction of Glycine with $\mathrm{Cu}(100)$ and $\mathrm{Cu}(111)$. Surf. Sci. 2003, 531, 304-318.

(20) Chen, Q.; Frankel, D. J.; Richardson, N. V. Chemisorption Induced Chirality: Glycine on $\mathrm{Cu}\{110\}$. Surf. Sci. 2002, 497, 37-46.

(21) Barlow, S. M.; Kitching, K. J.; Haq, S.; Richardson, N. V. A Study of Glycine Adsorption on a Cu110 Surface Using Reflection Absorption Infrared Spectroscopy. Surf. Sci. 1998, 401, 322-335.

(22) Han, J. W.; James, J. N.; Sholl, D. S. Chemical Speciation of Adsorbed Glycine on Metal Surfaces. J. Chem. Phys. 2011, 135, 034703.

(23) Rankin, R. B.; Sholl, D. S. Assessment of Heterochiral and Homochiral Glycine Adlayers on $\mathrm{Cu}(110)$ Using Density Functional Theory. Surf. Sci. 2004, 548, 301-308.

(24) Carravetta, V.; Monti, S.; Li, C.; Ågren, H. Theoretical Simulations of Structure and X-ray Photoelectron Spectra of Glycine and Diglycine Adsorbed on $\mathrm{Cu}(110)$. Langmuir 2013, 29, 10194-10204.

(25) Lawton, T. J.; Pushkarev, V.; Wei, D.; Lucci, F. R.; Sholl, D. S.; Gellman, A. J.; Sykes, E. C. H. Long Range Chiral Imprinting of $\mathrm{Cu}(110)$ by Tartaric Acid. J. Phys. Chem. C 2013, 117, 22290-22297.

(26) Karageorgaki, C.; Ernst, K.-H. A Metal Surface with Chiral Memory. Chem. Commun. (Camb). 2014, 50, 1814-1816.

(27) Wang, H.-S.; Wei, J.-P. Emerging Enantiomeric Resolution Materials with Homochiral Nano-fabrications. Nanoscale 2015, 7, 11815-11832.

(28) Gellman, A. J.; Tysoe, W. T.; Zaera, F. Surface Chemistry for Enantioselective Catalysis. Catal. Letters 2014, 145, 220-232. 
(29) Zheleva, Z. V.; Eralp, T.; Held, G. Complete Experimental Structure Determination of the $\mathrm{p}(3 \times 2)$ pg Phase of Glycine on $\mathrm{Cu}\{110\}$. J. Phys. Chem. C 2012, 116, 618-625.

(30) Chen, Q.; Richardson, N. V. Enantiomeric Interactions Between Nucleic Acid Bases and Amino Acids on Solid Surfaces. Nat. Mater. 2003, 2, 324-328.

(31) Bracco, G.; Holst, B. In Surface Science Techniques; Bracco, G., Holst, B., Eds.; Springer Series in Surface Sciences; Springer Berlin Heidelberg: Berlin, Heidelberg, 2013; Vol. 51.

(32) de la Figuera, J.; McCarty, K. F. Low-Energy Electron Microscopy. Surf. Sci. Tech. 2013, 531-561.

(33) Hohenberg, P.; Kohn, W. Inhomogeneous Electron Gas. Phys. Rev. 1964, 136, B864B871.

(34) Kohn, W.; Sham, L. J. Self-consistent Equations Including Exchange and Correlation Effects. Phys. Rev. 1965, 140, A1133-A1138.

(35) Wigner, E. P. The Transition State Method. Trans. Faraday Soc. 1938, 34, 29.

(36) Wigner, E. P. Some Remarks on the Theory of Reaction Rates. J. Chem. Phys. 1939, $7,646$.

(37) Michely, T.; Krug, J. Islands, Mounds and Atoms; Springer Series in Surface Sciences; Springer Berlin Heidelberg: Berlin, Heidelberg, 2004; Vol. 42; pp 1-286.

(38) Evansa, J.; Thiel, P.; Bartelt, M. Morphological Evolution During Epitaxial thin Film Growth: Formation of 2D Islands and 3D Mounds. Surf. Sci. Rep. 2006, 61, 1-128.

(39) Jansen, A. P. J. An Introduction to Kinetic Monte Carlo Simulations of Surface Reactions; Springer, 2012; p 254. 
(40) Binder, K.; Landau, D. P. Adv. Chem. Phys. Mol. Surf. Interact. Vol. 76; John Wiley \& Sons, Inc., 1989; pp 91-152.

(41) Liu, D.-J.; Evans, J. W. Realistic Multisite Lattice-gas Modeling and KMC Simulation of Catalytic Surface Reactions: Kinetics and Multiscale Spatial Behavior for COoxidation on Metal (1 0 0) Surfaces. Prog. Surf. Sci. 2013, 88, 393-521.

(42) Stamatakis, M.; Vlachos, D. G. Unraveling the Complexity of Catalytic Reactions via Kinetic Monte Carlo Simulation: Current Status and Frontiers. ACS Catal. 2012, 2, 2648-2663.

(43) Weinberg, W. H. Order-Disorder Phase Transitions in Chemisorbed Overlayers. Annu. Rev. Phys. Chem. 1983, 34, 217-243.

(44) Farkas, A.; Hess, F.; Over, H. Experiment-Based Kinetic Monte Carlo Simulations: CO Oxidation over $\mathrm{RuO}_{2}(110)$. J. Phys. Chem. C 2012, 116, 581-591.

(45) Merlet, C.; Forse, A. C.; Griffin, J. M.; Frenkel, D.; Grey, C. P. Lattice Simulation Method to Model Diffusion and NMR Spectra in Porous Materials. J. Chem. Phys. 2015, 142, 094701.

(46) Liu, D.-J.; Garcia, A.; Wang, J.; Ackerman, D. M.; Wang, C.-J.; Evans, J. W. ChemInform Abstract: Kinetic Monte Carlo Simulation of Statistical Mechanical Models and Coarse-Grained Mesoscale Descriptions of Catalytic Reaction-Diffusion Processes: 1D Nanoporous and 2D Surface Systems. ChemInform 2015, 46, no.

(47) Hopp, S. F.; Heuer, A. Kinetic Monte Carlo Study of Nucleation Processes on Patterned Surfaces. J. Chem. Phys. 2010, 133, 204101.

(48) Hopp, S. F.; Heuer, A. Anisotropic Behavior of Organic Molecules on Prepatterned Surfaces. J. Chem. Phys. 2012, 136, 154106. 
(49) Mues, T.; Heuer, A. Nucleation Growth: A Probabilistic Description of a Lattice Monte Carlo Simulation. Phys. Rev. B 2013, 88, 045411.

(50) Cuppen, H.; van Veenendaal, E.; van Suchtelen, J.; van Enckevort, W.; Vlieg, E. A Monte Carlo Study of Dislocation Growth and Etching of Crystals. J. Cryst. Growth 2000, 219, 165-175.

(51) Wales, D. J.; Hernández-Rojas, J. Exploring Energy Landscapes with Monte Carlo Methods. 2003, 690, 334-343.

(52) Middleton, T. F.; Wales, D. J. Comparison of Kinetic Monte Carlo and Molecular Dynamics Simulations of Diffusion in a Model Glass Former. J. Chem. Phys. 2004, 120, 8134-8143.

(53) Hernández-Rojas, J.; Wales, D. J. Supercooled Lennard-Jones Liquids and Glasses: a Kinetic Monte Carlo Approach. J. Non. Cryst. Solids 2004, 336, 218-222.

(54) Matera, S.; Blomberg, S.; Hoffmann, M. J.; Zetterberg, J.; Gustafson, J.; Lundgren, E.; Reuter, K. Evidence for the Active Phase of Heterogeneous Catalysts through In Situ Reaction Product Imaging and Multiscale Modeling. ACS Catal. 2015, 5, 4514-4518.

(55) Knapp, B.; Demharter, S.; Deane, C. M.; Minary, P. Exploring Peptide/MHC Detachment Processes Using Hierarchical Natural Move Monte Carlo. Bioinformatics 2016, 32, 181-186.

(56) Herman, M. F. Improving the Efficiency of Monte Carlo Surface Hopping Calculations. J. Phys. Chem. B 2014, 118, 8026-8033.

(57) Reuter, K.; Scheffler, M. First-principles Kinetic Monte Carlo Simulations for Heterogeneous Catalysis: Application to the $\mathrm{CO}$ Oxidation at $\mathrm{Ru} \mathrm{O} 2(110)$. Phys. Rev. B 2006, 73, 045433. 
(58) Rogal, J.; Reuter, K.; Scheffler, M. CO Oxidation on Pd(100) at Technologically Relevant Pressure Conditions: First-principles Kinetic Monte Carlo Study. Phys. Rev. B 2008, 77, 155410.

(59) Xu, L.; Campbell, C. T.; Jónsson, H.; Henkelman, G. Kinetic Monte Carlo Simulations of Pd Deposition and Island Growth on $\mathrm{MgO}(100)$. Surf. Sci. 2007, 601, 3133-3142.

(60) Xu, L.; Mei, D.; Henkelman, G. Adaptive Kinetic Monte Carlo Simulation of Methanol Decomposition on $\mathrm{Cu}(100)$. J. Chem. Phys. 2009, 131, 244520.

(61) Stamatakis, M.; Vlachos, D. G. Unraveling the Complexity of Catalytic Reactions via Kinetic Monte Carlo Simulation: Current Status and Frontiers. ACS Catal. 2012, 2, 2648-2663.

(62) Hess, F.; Over, H. Kinetic Monte Carlo Simulations of Heterogeneously Catalyzed Oxidation Reactions. Catal. Sci. Technol. 2014, 4, 583.

(63) Hoffmann, M. J.; Scheffler, M.; Reuter, K. Multi-lattice Kinetic Monte Carlo Simulations from First Principles: Reduction of the $\operatorname{Pd}(100)$ Surface Oxide by CO. ACS Catal. 2015, 5, 1199-1209.

(64) Wales, D. J. Discrete Path Sampling. Mol. Phys. 2002, 100, 3285-3305.

(65) Trygubenko, S. A.; Wales, D. J. Kinetic Analysis of Discrete Path Sampling Stationary Point Databases. Mol. Phys. 2006, 104, 1497-1507.

(66) Wales, D. J. Energy Landscapes: Calculating Pathways and Rates. Int. Rev. Phys. Chem. 2006, 25, 237-282.

(67) Wales, D. J. Calculating Rate Constants and Committor Probabilities for Transition Networks by Graph Transformation. J. Chem. Phys. 2009, 130, 204111. 
(68) Schütte, C.; Walter, J.; Hartmann, C.; Huisinga, W. An Averaging Principle for Fast Degrees of Freedom Exhibiting Long-Term Correlations. Multiscale Model. Simul. 2004, 2, 501-526.

(69) Melnik, R. V. Markov Chain Network Training and Conservation Law Approximations: Linking Microscopic and Macroscopic Models for Evolution. Appl. Math. Comput. 2008, 199, 315-333.

(70) Sarich, M.; Noé, F.; Schütte, C. On the Approximation Quality of Markov State Models. Multiscale Model. Simul. 2010, 8, 1154-1177.

(71) Prinz, J.-H.; Wu, H.; Sarich, M.; Keller, B.; Senne, M.; Held, M.; Chodera, J. D.; Schütte, C.; Noé, F. Markov Models of Molecular Kinetics: Generation and Validation. J. Chem. Phys. 2011, 134, 174105.

(72) Nüske, F.; Keller, B. G.; Pérez-Hernández, G.; Mey, A. S. J. S.; Noé, F. Variational Approach to Molecular Kinetics. J. Chem. Theory Comput. 2014, 10, 1739-1752.

(73) Fichthorn, K. A.; Weinberg, W. H. Theoretical Foundations of Dynamical Monte Carlo Simulations. J. Chem. Phys. 1991, 95, 1090.

(74) Voter, A. F. Classically Exact Overlayer Dynamics: Diffusion of Rhodium Clusters on Rh(100). Phys. Rev. B 1986, 34, 6819-6829.

(75) Snurr, R. Q.; Bell, A. T.; Theodorou, D. N. Investigation of the Dynamics of Benzene in Silicalite Using Transition-State Theory. J. Phys. Chem. 1994, 98, 11948-11961.

(76) Henkelman, G.; Jónsson, H. Long Time Scale Kinetic Monte Carlo Simulations Without Lattice Approximation and Predefined Event Table. J. Chem. Phys. 2001, 115, 9657.

(77) Stamatakis, M. Kinetic Modelling of Heterogeneous Catalytic Systems. J. Phys. Condens. Matter 2015, 27, 013001. 
(78) Voter, A. F.; Montalenti, F.; Germann, T. C. Extending the Time Scale in Atomistic Simulations of Materials. Annu. Rev. Mater. Res. 2002, 32, 321-346.

(79) Terrell, R.; Welborn, M.; Chill, S. T.; Henkelman, G. Database of Atomistic Reaction Mechanisms with Application to Kinetic Monte Carlo. J. Chem. Phys. 2012, 137, 014105 .

(80) Salciccioli, M.; Stamatakis, M.; Caratzoulas, S.; Vlachos, D. A Review of Multiscale Modeling of Metal-catalyzed Reactions: Mechanism Development for Complexity and Emergent Behavior. Chem. Eng. Sci. 2011, 66, 4319-4355.

(81) Xu, L.; Henkelman, G. Adaptive Kinetic Monte Carlo for First-Principles Accelerated Dynamics. J. Chem. Phys. 2008, 129, 114104.

(82) Trushin, O.; Karim, A.; Kara, A.; Rahman, T. S. Self-learning Kinetic Monte Carlo Method: Application to $\mathrm{Cu}(111)$. Phys. Rev. B 2005, 72, 115401.

(83) El-Mellouhi, F.; Mousseau, N.; Lewis, L. J. Kinetic Activation-Relaxation Technique: an Off-lattice Self-learning Kinetic Monte Carlo Algorithm. Phys. Rev. B 2008, 78, 153202.

(84) Whitelam, S.; Jack, R. L. The Statistical Mechanics of Dynamic Pathways to SelfAssembly. Annu. Rev. Phys. Chem. 2015, 66, 143-163.

(85) Béland, L. K.; Brommer, P.; El-Mellouhi, F.; Joly, J.-F.; Mousseau, N. Kinetic Activation-Relaxation Technique. Phys. Rev. E. Stat. Nonlin. Soft Matter Phys. 2011, 84,046704 .

(86) Pedersen, A.; Jónsson, H. Distributed Implementation of the Adaptive Kinetic Monte Carlo Method. Math. Comput. Simul. 2010, 80, 1487-1498. 
(87) Pogodin, S.; López, N. A More Accurate Kinetic Monte Carlo Approach to a Monodimensional Surface Reaction: The Interaction of Oxygen with the RuO2(110) Surface. ACS Catal. 2014, 4, 2328-2332.

(88) Mousseau, N.; Béland, L. K.; Brommer, P.; El-Mellouhi, F.; Joly, J.-F.; N'Tsouaglo, G. K.; Restrepo, O.; Trochet, M. Following Atomistic Kinetics on Experimental Timescales with the Kinetic Activation-Relaxation Technique. Comput. Mater. Sci. 2015, 100, 111-123.

(89) Kunz, L.; Kuhn, F. M.; Deutschmann, O. Kinetic Monte Carlo Simulations of Surface Reactions on Supported Nanoparticles: a Novel Approach and Computer Code. J. Chem. Phys. 2015, 143, 044108.

(90) Chill, S. T.; Henkelman, G. Molecular Dynamics Saddle Search Adaptive Kinetic Monte Carlo. J. Chem. Phys. 2014, 140, 214110.

(91) Gillespie, D. T. A General Method for Numerically Simulating the Stochastic Time Evolution of Coupled Chemical Reactions. J. Comput. Phys. 1976, 22, 403-434.

(92) Sacchi, M.; Wales, D. J.; Jenkins, S. J. Energy Landscapes and Dynamics of Glycine on $\mathrm{Cu}(110)$. Phys. Chem. Chem. Phys. 2017, 19, 16600-16605.

(93) Rankin, R. B.; Sholl, D. S. Structures of Glycine, Enantiopure Alanine, and Racemic Alanine Adlayers on $\mathrm{Cu}(110)$ and $\mathrm{Cu}(100)$ Surfaces. J. Phys. Chem. B 2005, 109, 16764-16773.

(94) Feyer, V.; Plekan, O.; Tsud, N.; Lyamayev, V.; Cháb, V.; Matolín, V.; Prince, K. C.; Carravetta, V. Adsorption Structure of Glycyl-Glycine on $\mathrm{Cu}(110)$. J. Phys. Chem. C 2010, 114, 10922-10931.

(95) Zhang, Y. Y.; Wang, Y.-L.; Meng, L.; Zhang, S. B.; Gao, H.-J. Thermally Controlled 
Adenine Dimer Chain Rotation on $\mathrm{Cu}(110)$ : The Critical Role of van der Waals Interactions. J. Phys. Chem. C 2014, 118, 6278-6282.

(96) Eralp, T.; Shavorskiy, A.; Zheleva, Z. V.; Held, G.; Kalashnyk, N.; Ning, Y.; Linderoth, T. R. Global and Local Expression of Chirality in Serine on the $\mathrm{Cu}\{110\}$ Surface. Langmuir 2010, 26, 18841-18851.

(97) Strodel, B.; Wales, D. J. Free Energy Surfaces from an Extended Harmonic Superposition Approach and Kinetics for Alanine Dipeptide. Chem. Phys. Lett. 2008, 466, $105-115$.

(98) Neelamraju, S.; Oakley, M. T.; Johnston, R. L. Chiral Effects on Helicity Studied via the Energy Landscape of Short (d, l)-Alanine Peptides. J. Chem. Phys. 2015, 143, 165103.

(99) Monti, S.; Carravetta, V.; Li, C.; Ågren, H. A Computational Study of the Adsorption and Reactive Dynamics of Diglycine on $\mathrm{Cu}(110)$. J. Phys. Chem. C 2014, 118, 36103619 .

(100) Nyberg, M.; Odelius, M.; Nilsson, A.; Pettersson, L. G. M. Hydrogen Bonding Between Adsorbed Deprotonated Glycine Molecules on $\mathrm{Cu}(110)$. J. Chem. Phys. 2003, 119, 12577.

(101) Vlachos, D. G. Temporal Coarse-Graining of Microscopic-lattice Kinetic Monte Carlo Simulations via $\tau$-leaping. Phys. Rev. E 2008, 78, 046713.

(102) Rommel, J. B. Tau Leaping for Molecular Kinetics of Glycine Clusters on Cu(110). http://doi.org/10.5281/zenodo.159466 2016, Fortran code.

(103) Clark, S. J.; Segall, M. D.; Pickard, C. J.; Hasnip, P. J.; Probert, M. J.; Refson, K.; Payne, M. C. First Principles Methods Using CASTEP. Z. Kristallogr. 2011, 220, $567-570$. 
(104) Monkhorst, H. J.; Pack, J. D. Special Points for Brillouin-zone Integrations. Phys. Rev. B 1976, 13, 5188.

(105) Sacchi, M.; Jenkins, S. J. Co-adsorption of Water and Glycine on $\mathrm{Cu}\{110\}$. Phys. Chem. Chem. Phys. 2014, 16, 6101.

(106) Perdew, J. P.; Burke, K.; Ernzerhof, M. Generalized Gradient Approximation Made Simple. Phys. Rev. Lett. 1996, 77, 3865-3868.

(107) Vanderbilt, D. Soft Self-consistent Pseudopotentials in a Generalized Eigenvalue Formalism. Phys. Rev. B 1990, 41, 7892.

(108) Govind, N.; Petersen, M.; Fitzgerald, G.; King-Smith, D.; Andzelm, J. A Generalized Synchronous Transit Method for Transition State Location. Comp. Mat. Sci. 2003, 28, 250-258.

(109) Kumeda, Y.; Wales, D. J.; Munro, L. J. Transition States and Tearrangement Mechanisms from Hybrid Eigenvector-following and Density Functional Theory: Application to $\mathrm{C}_{10} \mathrm{H}_{10}$ and Defect Migration in Crystalline Silicon. Chem. Phys. Lett. 2001, 341, 185-194.

(110) Wales, D. J. Energy Landscapes; Cambridge University Press, Cambridge, 2003.

(111) Munro, L. J.; Wales, D. J. Defect Migration in Crystalline Silicon. Phys. Rev. B 1999, $59,3969$.

(112) Haq, S.; Massey, A.; Moslemzadeh, N.; Robin, A.; Barlow, S. M.; Raval, R. Racemic Versus Enantiopure Alanine on $\mathrm{Cu}(110)$ : an Experimental Study. Langmuir 2007, 23, 10694-10700.

(113) Toomes, R.; Kang, J.; Woodruff, D.; Polcik, M.; Kittel. M.,; Hoeft, J.-T. Can Glycine form Homochiral Structural Domains on low-index Copper Surfaces? Surf. Sci. 2003, 522, L9-L14. 
(114) Eralp, T.; Shavorskiy, A.; Zheleva, Z. V.; Dhanak, V. R.; Held, G. Hydrogen Bondinduced Pair Formation of Glycine on the Chiral $\mathrm{Cu}\{531\}$ Surface. Langmuir 2010, 26, 10918-10923.

(115) Yun, Y.; Wei, D.; Sholl, D. S.; Gellman, A. J. Equilibrium Adsorption of d- and lAlanine Mixtures on Naturally Chiral $\mathrm{Cu}\{3,1,17\}$ R\&S Surfaces. J. Phys. Chem. C 2014, 118, 14957-14966.

(116) Meskine, H.; Matera, S.; Scheffler, M.; Reuter, K.; Metiu, H. Examination of the Concept of Degree of Rate Control by First-principles Kinetic Monte Carlo Simulations. Surf. Sci. 2009, 603, 1724-1730.

(117) Núñez, M.; Vlachos, D. G. Steady State Likelihood Ratio Sensitivity Analysis for Stiff Kinetic Monte Carlo Simulations. J. Chem. Phys. 2015, 142, 044108.

(118) Forster, M.; Dyer, M. S.; Persson, M.; Raval, R. Tailoring Homochirality at Surfaces: Going Beyond Molecular Handedness. J. Am. Chem. Soc. 2011, 133, 15992-6000. 


\section{Graphical TOC Entry}

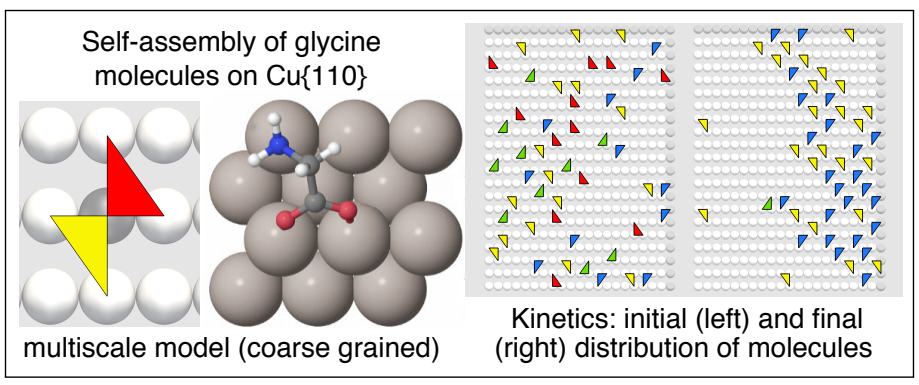

